\title{
Measuring atmospheric neutrino oscillations with neutrino telescopes
}

\author{
Ivone F. M. Albuquerque* \\ Department of Astronomy \& Space Sciences Laboratory, University of California, Berkeley, California 94720 \\ George F. Smoot ${ }^{\dagger}$ \\ Department of Physics, Lawrence Berkeley National Laboratory \& Space Sciences Laboratory, University of California, \\ Berkeley, California 94720
}

(Received 29 March 2001; published 8 August 2001)

\begin{abstract}
Neutrino telescopes with large detection volumes can demonstrate whether the current indications of neutrino oscillation are correct or if a better description can be achieved with nonstandard alternatives. Observations of contained muons produced by atmospheric neutrinos can better constrain the allowed region for oscillations or determine the relevant parameters of nonstandard models. We analyze the possibility of neutrino telescopes measuring atmospheric neutrino oscillations. We suggest adjustments to improve this potential. An addition of four densely instrumented strings to the AMANDA II detector makes oscillation observations feasible. Such a configuration is competitive with current and proposed experiments.
\end{abstract}

DOI: 10.1103/PhysRevD.64.053008

PACS number(s): 14.60.Pq

\section{INTRODUCTION}

Experimental observations (Super-Kamiokande [1], Kamiokande [2], IMB [3] and Soudan [4]) for atmospheric muon and electron neutrinos have found that the ratio of the number of these neutrino species does not agree with theoretical prediction. All experiments measuring the flux of solar neutrinos observe a deficit compared with the solar model predictions [5]. The ratio of muon to electron events observed in atmospheric neutrino interactions is measured by most experiments to be less than expected from models of cosmic ray interactions in the atmosphere [6]. Neutrino oscillation can explain these results. The measurement of the up-down asymmetry of this ratio by the Super-Kamionkande Collaboration [1] is generally considered to be the strongest evidence for neutrino oscillations. As oscillations would most likely imply that neutrinos have mass, many researchers have fitted the available data for the mass difference among different neutrino species.

While the cumulative evidence for neutrino oscillations is very striking, a definitive proof that the observed anomalies are actually due to neutrino oscillations is still missing. In particular the current observations of atmospheric neutrinos are consistent with the hypothesis of maximal $\nu_{\mu}$ oscillations, but do not exclude some alternative unconventional explanations, such as neutrino decay [7], micron-scale extra dimensions [8] or quantum decoherence in propagation [9].

Cosmic rays traveling to Earth will interact with its atmospheric nuclei. These interactions produce hadrons which decay eventually into atmospheric neutrinos. Up to neutrino energies of about $100 \mathrm{GeV}$, the main result from these interactions is pion production $\pi^{+}\left(\pi^{-}\right)$. These will decay into $\mu^{+}+\nu_{\mu}\left(\mu^{-}+\bar{\nu}_{\mu}\right)$ almost $100 \%$ of the time. The secondary

\footnotetext{
*Electronic mail: IFAlbuquerque@lbl.gov

†Electronic mail: GFSmoot@lbl.gov
}

$\mu^{+}\left(\mu^{-}\right)$will decay into $e^{+}+\nu_{e}+\bar{\nu}_{\mu}\left(e^{-}+\bar{\nu}_{e}+\nu_{\mu}\right)$ which will give a $\nu_{\mu}+\bar{\nu}_{\mu}$ to $\nu_{e}+\bar{\nu}_{e}$ ratio (r) of 2:1.

However, depending on the cosmic ray energy and where it interacts in the Earth's atmosphere, the muon might not decay before reaching the ground and corrections to this ratio have to be included. Another effect to be included is the Earth's magnetic field action on lower energy particles. These effects have been modeled and simulated and one looks for a ratio $(R)$ that relates $r_{\text {exp }}$ from experimental data to $r_{\text {sim }}$ from simulations $\left(R=r_{\text {exp }} / r_{\text {sim }}\right)$. In this way one can reduce the bias introduced by uncertainties in understanding these effects.

Super-Kamiokande, Kamiokande, IMB and Soudan experiments [1-4] found the ratio $\mathrm{R}$ to be lower than expected, with fewer $\nu_{\mu}$ 's. Frejus and Nusex experiments $[10,11]$ have not found any anomaly in R.

If neutrinos have mass, one type of neutrino can oscillate into another. The probability $(\mathrm{P})$ for atmospheric neutrino oscillations, in a two neutrino mixing scenario, is given by [12]

$$
P=\sin ^{2} 2 \theta \sin ^{2}\left(1.27 \frac{L}{E} \Delta m^{2}\right)
$$

where $\theta$ is the mixing angle, $\Delta m^{2}$ is the difference of the squared mass for two types of neutrinos in $\mathrm{eV}^{2}, L$ is the distance traveled by the original neutrino in $\mathrm{km}$ and $E$ is the neutrino energy in $\mathrm{GeV}$.

The Super-Kamiokande analysis [1] constrains $\Delta m^{2}$ from $5 \times 10^{-4}$ to $6 \times 10^{-3} \mathrm{eV}^{2}$ and $\theta$ to greater than 0.82 at $90 \%$ confidence level. The most probable solution is $\Delta m^{2}$ $=3.5 \times 10^{-3} \mathrm{eV}^{2}$ and $\sin ^{2} 2 \theta=1$ (full mixing). Also the oscillation of a muon neutrino into a tau neutrino is favored over an oscillation into a sterile neutrino [13].

If the atmospheric neutrino result is joined with the solar and short baseline beam measurements, they indicate evidence of a non-zero $\Delta m^{2}$. However, new neutrino oscillation 


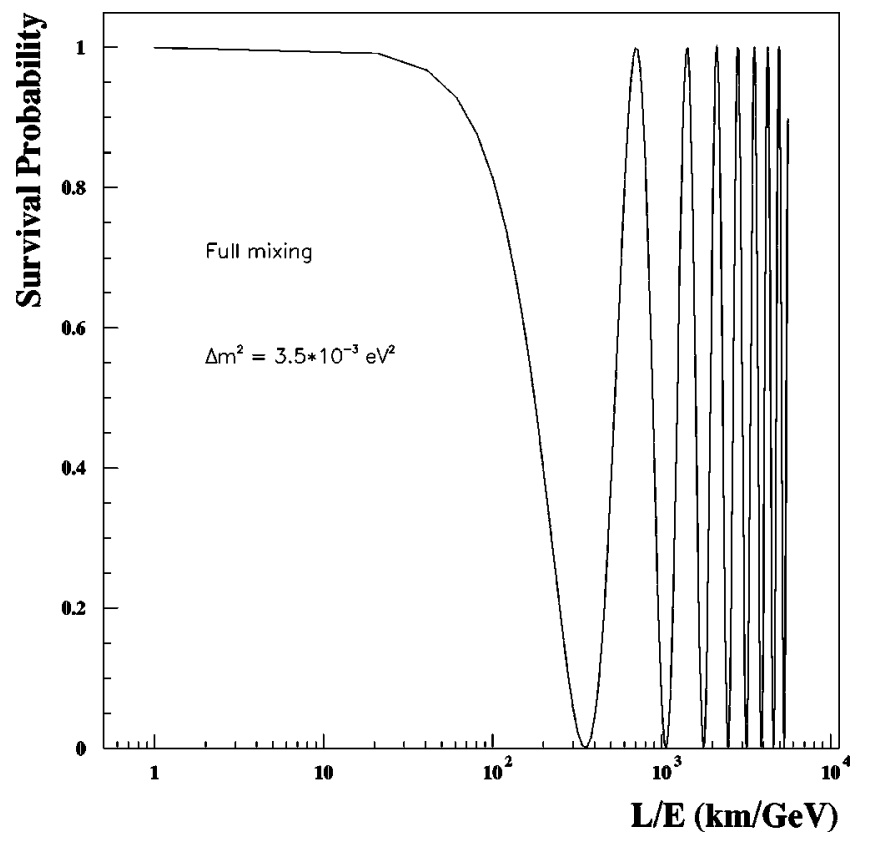

FIG. 1. Neutrino survival probability versus $L / E$. Full neutrino mixing $\left(\sin ^{2} 2 \theta=1\right)$ and $\Delta m^{2}=3.5 \times 10^{-3} \mathrm{eV}^{2}$ is assumed.

experiments are needed to precisely measure $\Delta m^{2}$ as well as to decide among experimental results that are in disagreement. As an example, the Super-Kamiokande [1] results barely overlap with Kamiokande [2] results.

As the detector area and volume covered by current or proposed high-energy astrophysical neutrino telescopes are large compared to underground detectors, it is possible for these experiments to contribute to the understanding of atmospheric neutrino oscillations. In this paper we investigate the possibility of AMANDA [14] and IceCube [15] detectors measuring atmospheric neutrino oscillations. Similar analyses would apply for the ANTARES [16] and NESTOR [17] detectors.

\section{MEASUREMENT OF ATMOSPHERIC OSCILLATIONS}

Figure 1 shows the neutrino flavor survival probability $\left(P_{S}\right)$ versus the ratio of the distance traveled by the neutrino and its energy $(L / E)$. This probability is given by $1-P_{\sim}$ where $P \sim$ is the neutrino oscillation probability given in Eq. (1).

As one can experimentally determine the number of events with energy $E$ as a function of the zenith angle $\left(\theta_{Z}\right)$, a plot of the survival probability versus $\cos \theta_{Z}$ provides a more convenient indication of what could be accomplished by neutrino telescopes.

Equation (1) shows that the larger the distance the neutrino travels, the greater the possibility of oscillation. For energies of $10 \mathrm{GeV}$ and above only upward going neutrinos, those which come from below the detector after crossing the Earth, will have a significant probability of oscillating.

We define $\theta_{Z}$ as the angle from the detector axis (defined by a line from the detector to the center of the Earth) to the direction of the particle arrival at the detector [33]. For a

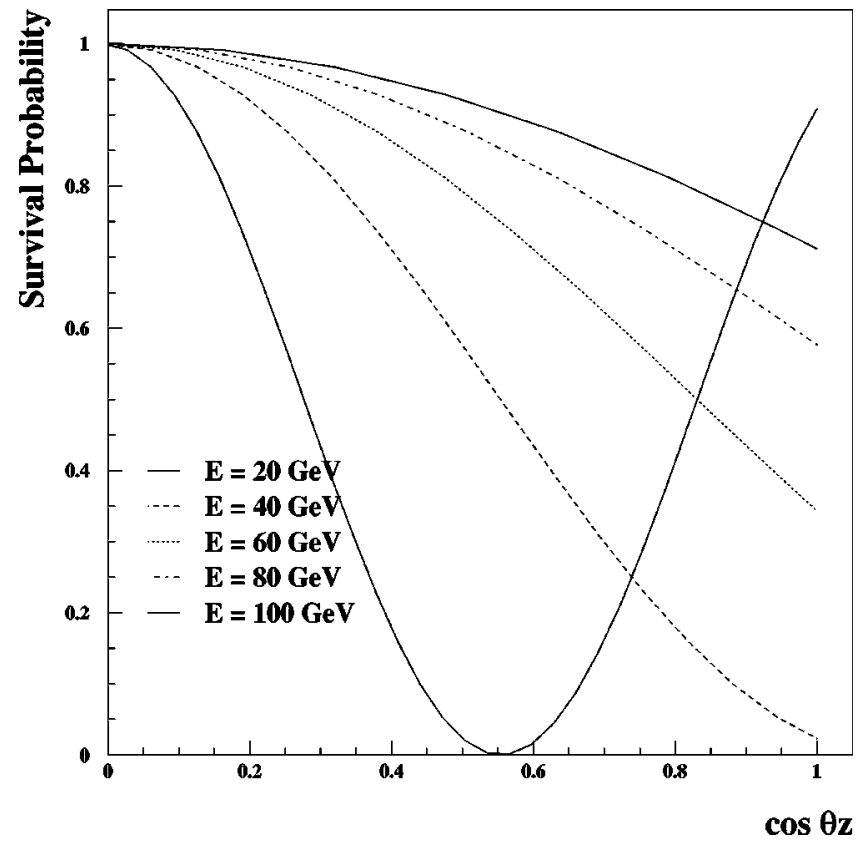

FIG. 2. Neutrino survival probability versus cosine of the zenith angle for different neutrino energies as labeled. Full neutrino mixing and $\Delta m^{2}=3.5 \times 10^{-3} \mathrm{eV}^{2}$ is assumed. The probability is given for an idealized detector $2 \mathrm{~km}$ deep in the Earth. It indicates that a neutrino telescope needs a low energy threshold (less than $30 \mathrm{GeV}$ ) to be able to measure atmospheric oscillations well.

detector located at a distance $R_{d}$ from the center of the Earth, the distance traveled $(L)$ by the neutrino will be

$$
L=R_{d} \cos \theta_{Z}+\sqrt{R_{\nu}^{2}-R_{d}^{2}+R_{d}^{2} \cos ^{2} \theta_{Z}}
$$

where $R_{\nu}$ is the radius from the Earth's center at which the neutrino is produced, $R_{\nu} \simeq R_{\oplus}+15 \mathrm{~km}$, where $R_{\oplus}$ is the Earth radius. This equation reduces to $L=R_{d}+R_{\nu} \sim 2 R_{\oplus}$ for $\cos \theta_{Z}=1$ (vertical upcoming neutrinos), to $L=\sqrt{R_{\nu}^{2}-R_{d}^{2}}$ for $\cos ^{2} \theta_{Z}=0$ (horizontal neutrinos), and $L=R_{\nu}-R_{d} \sim 15 \mathrm{~km}$ for $\cos \theta_{Z}=-1$ (vertical downgoing neutrinos).

At the energies we are considering (10-100 GeV) only upwards going neutrinos will have a significant probability of oscillating. Thus the neutrino production point in the atmosphere will not be significant when compared to the length traveled through the Earth.

Figure 2 shows $\cos \theta_{Z}$ versus the survival probability as a function of energy. We assume that the detector is $2 \mathrm{~km}$ deep in the Earth (ice or water). It can be seen that it is easier to detect oscillations using lower energy neutrinos. Therefore to measure neutrino oscillations the telescope energy threshold cannot be too high and the energy resolution must be good. However, for high-energy neutrino telescopes the sensing elements are placed far apart to gain detector volume and the trigger energy threshold is usually set high to avoid atmospheric muon and neutrino background. The detector design has to be optimized, if it is to be used for both of these two types of observations. 

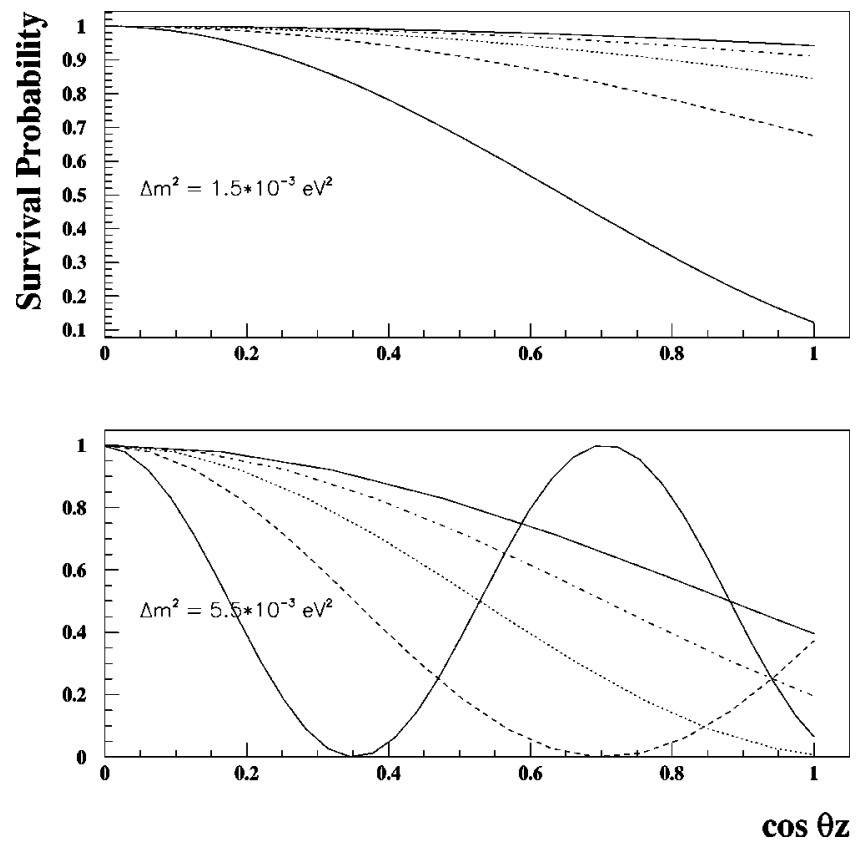

FIG. 3. Neutrino survival probability versus cosine of the zenith angle for different neutrino energies (as labeled in Fig. 2). The probability is given for a detector $2 \mathrm{~km}$ deep in the Earth. Full mixing is assumed and $\Delta m^{2}=1.5 \times 10^{-3}, \Delta m^{2}=5.5 \times 10^{-3} \mathrm{eV}^{2}$ as labeled. One can see that even for larger $\Delta m^{2}$ the neutrino telescope energy threshold has to be equal or less than $30 \mathrm{GeV}$.

Both Figs. 1 and 2 assume full mixing and $\Delta m^{2}=3.5$ $\times 10^{-3} \mathrm{eV}^{2}$. In Fig. 3 we show the same dependencies as in Fig. 2, but for different values of $\Delta m^{2}$. From this figure one can see that, depending on the energy threshold, it may be possible to better constrain $\Delta m^{2}$ with a neutrino telescope. To understand whether or not this measurement can be done, one has to take into account the atmospheric neutrino flux, the rate of contained events and the energy resolution of the detector.

Figure 4 shows the neutrino oscillation pattern for $20 \mathrm{GeV}$ neutrinos superimposed by other possible explanations which are compatible with the Superkamiokande [1] results. Measuring this oscillation pattern can allow to distinguish among different scenarios for the muon neutrino deficit.

\section{ATMOSPHERIC NEUTRINO FLUX}

To determine the number of events expected to be detected, one has to convolve the survival probability with the atmospheric neutrino flux. We determine this flux versus $\cos \theta_{Z}$ from the flux calculated by Volkova [18] and compare it with other calculations $[19,20]$.

Volkova derives the atmospheric neutrino flux from the decay of light mesons $(K, \pi)$ and $\mu$ 's and from the decay of short-lived particles (prompt decay) which mainly consist of charm particles. The latter will only be significant at higher energies (around a PeV) but we include it for completeness.

The differential energy spectra of atmospheric muon neutrinos from light mesons and muons can be approximated by [34]

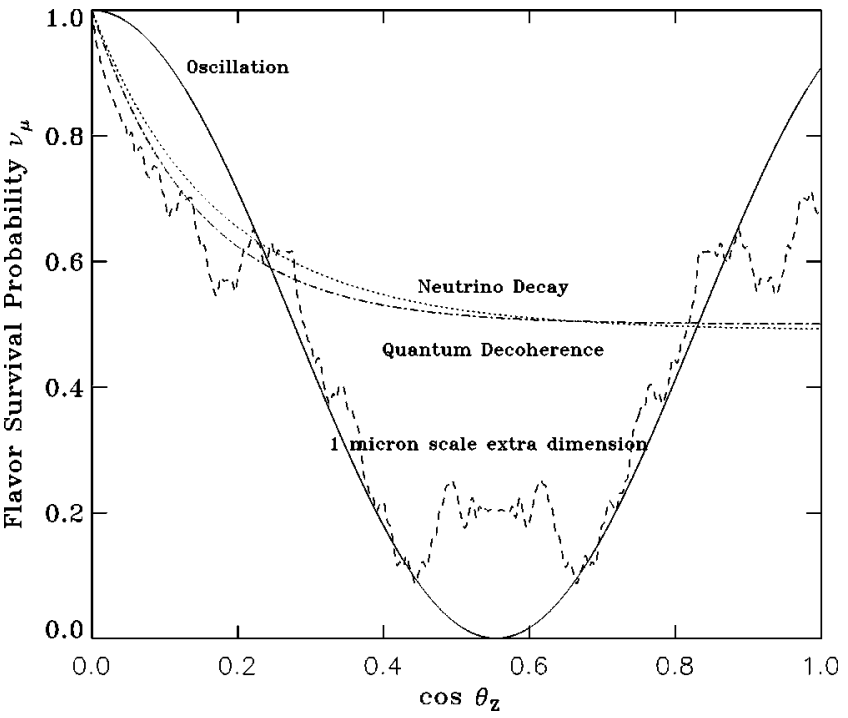

FIG. 4. Four theoretical scenarios that are consistent with the Super-Kamionkande results. (1) Solid line shows best fitted standard neutrino oscillations. Neutrino survival probability versus $\cos \theta_{Z}$ for $20 \mathrm{GeV}$ neutrinos crossing through the Earth $(L=$ Earth diameter). Full neutrino mixing and $\Delta m^{2}=3.5 \times 10^{-3} \mathrm{eV}^{2}$ is assumed. (2) Dashed line is effect of additional dimension with characteristic radius of about a micron [8]. (3) Dotted line is effect of a decaying neutrino [7]. (4) Dashed dotted line is the effect of quantum decoherence [9].

$$
\begin{aligned}
&\left(\frac{d N}{d E}\right)_{\text {light }}= 2.85 \times 10^{-2} E^{-2.69}\left(\frac{1}{1+6 E / E_{\pi}\left(\theta_{Z}\right)}\right. \\
&\left.+\frac{0.213}{1+1.44 E / E_{k}\left(\theta_{Z}\right)}\right), \\
& 10 \leqslant E<5.4 \times 10^{5} \mathrm{GeV} \\
&=0.48 E^{-4.04}\left(E_{\pi}\left(\theta_{Z}\right)+0.89 E_{K}\left(\theta_{Z}\right)\right), \\
& E \geqslant 5.4 \times 10^{5} \mathrm{GeV}, \text { (3) }
\end{aligned}
$$

where $E_{\pi}\left(\theta_{Z}\right)$ and $E_{K}\left(\theta_{Z}\right)$ are the $\pi$ and $K$ critical energies, $E$ is the neutrino energy and the spectra is given in units of neutrinos per $\mathrm{cm}^{2} \mathrm{sec} \mathrm{sr} \mathrm{GeV}$. The critical energy is the one for which the probability for a nuclear interaction in one nuclear mean free path equals the decay probability in the same path. It depends on the zenith angle since the atmosphere density varies with depth and horizontal events cross more dense regions than the vertical ones. $[18]$

The spectra for prompt neutrinos can be approximated by

$$
\begin{aligned}
\left(\frac{d N}{d E}\right)_{\text {prompt }} & =2.4 \times 10^{-5} E^{-2.65}, \quad E<2.3 \times 10^{6} \mathrm{GeV} \\
& =3.9 \times 10^{-3} E^{-3}, \quad E \geqslant 2.3 \times 10^{6} \mathrm{GeV} .
\end{aligned}
$$

In Fig. 5 we show the vertical and horizontal neutrino energy spectra and the contributions from $K, \pi$, and $\mu$ 's and 


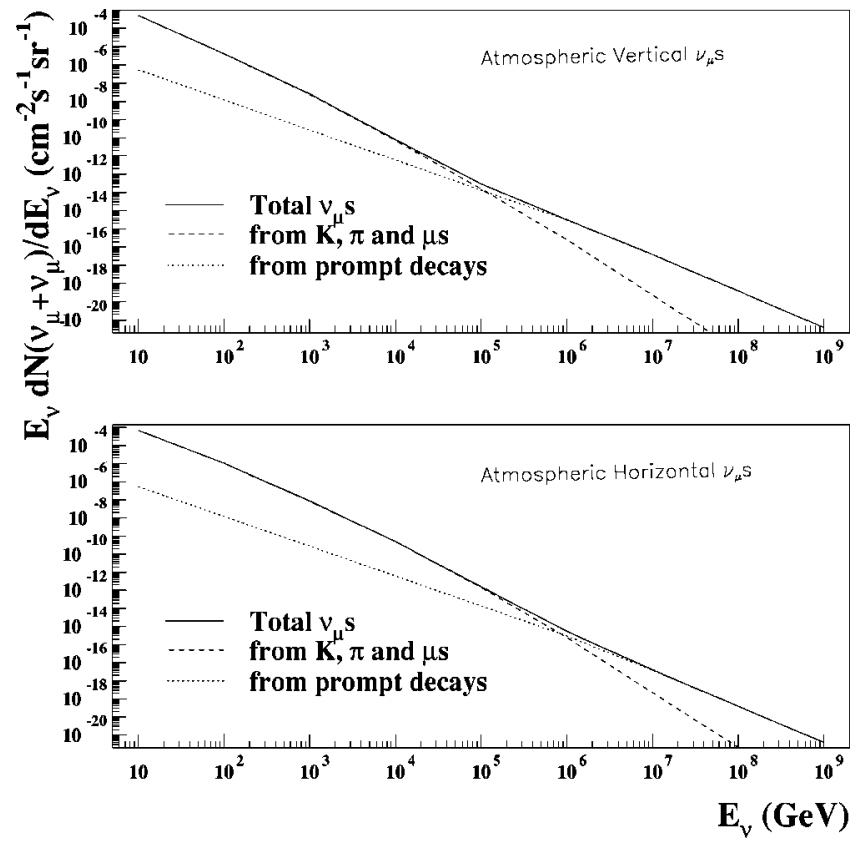

FIG. 5. Atmospheric vertical and horizontal (as labeled) muon neutrino energy spectra based on [18]. Also shown is the contribution from $K, \pi$, and $\mu$ 's and from prompt decays.

from prompt decays. One can see that the prompt contribution is negligible at lower energies. In Fig. 6 we expand the lower energy region and plot the total flux for vertical $\left(\cos \theta_{Z}=1\right)$ and horizontal $\left(\cos \theta_{Z}=0\right)$ spectra. As expected the number of horizontal neutrinos is slightly higher.

We compare this flux with that obtained by [19] and [20].

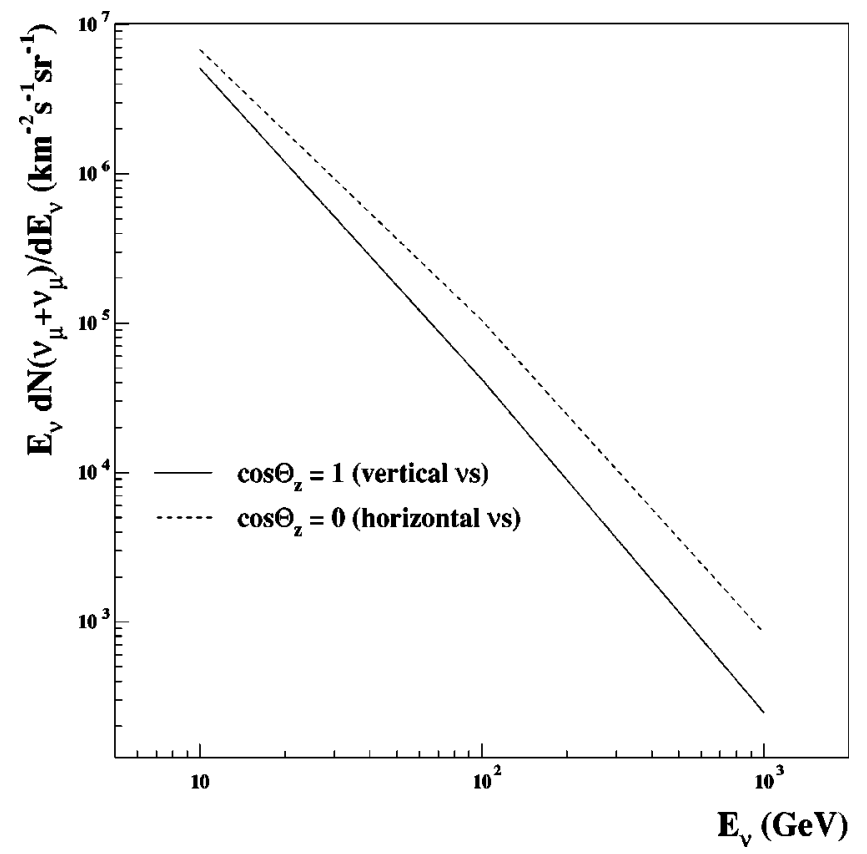

FIG. 6. Atmospheric vertical and horizontal (as labeled) muon neutrino energy spectra. Note that it is plotted per $\mathrm{km}^{2}$ instead of $\mathrm{cm}^{2}$ as in the previous figure. The flux of horizontal neutrinos is higher than vertically down going neutrinos since the muons have more opportunity to decay.

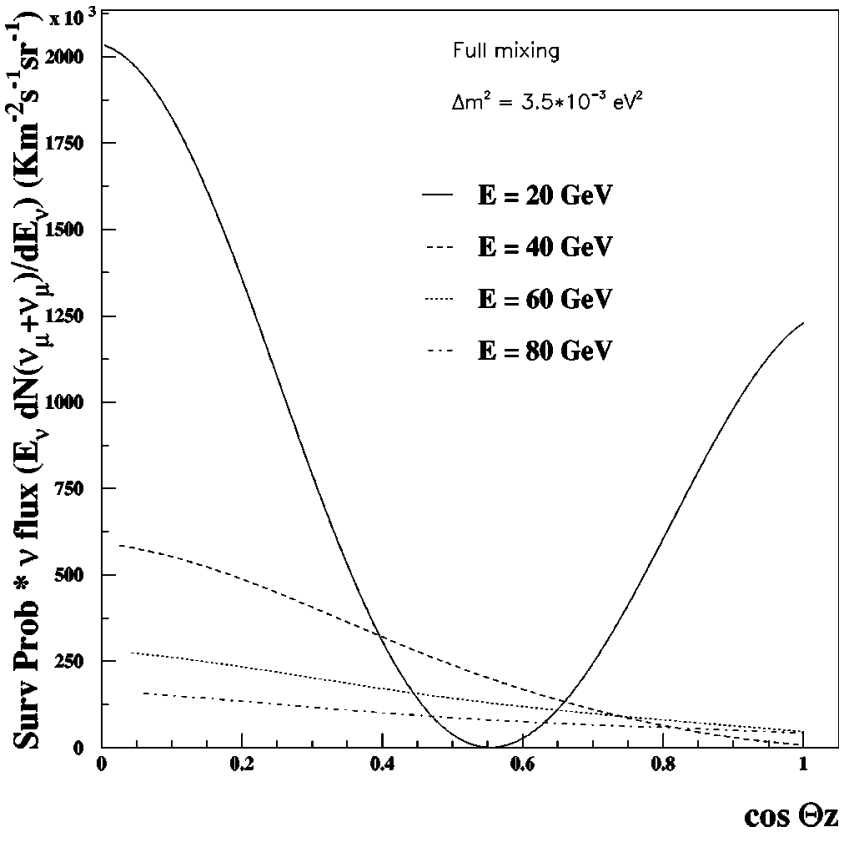

FIG. 7. Muon neutrino survival probability weighted by atmospheric muon neutrino energy spectrum. As expected, neutrino oscillations are enhanced at lower energies by a combination of higher flux and more rapid oscillation probability.

The largest discrepancy between these calculations is of about 15\% (see Fig. 14 of Ref. [19] and Fig. 7 of Ref. [20]). In the energy range of interest to our work, the Volkova spectrum is an underestimate compared to these other spectra. We will use the atmospheric neutrino flux based on the Volkova spectra. This is a more conservative spectrum and having a larger flux will only enhance the possibility of measuring neutrino oscillations. Also important is to compare the slope of these spectra and Fig. 7 of Ref. [20] shows that for energies between 10 and $100 \mathrm{GeV}$ the difference between the slope of the Volkova spectrum and of the spectrum determined in [20] or [19] is at maximum $1 \%$.

We now proceed to convolve the survival probability for upward going neutrinos shown in Fig. 2 by the flux obtained from the above analysis. This is shown in Fig. 7.

As expected, neutrino oscillations are enhanced at lower energies.

\section{CONTAINED EVENTS}

When muon neutrinos undergo a charged current interaction, they produce a muon. This will propagate losing energy and eventually comes to rest and decays. If the entire event happens inside the detector (a "contained" event) it is much easier to reconstruct the event than if it is not fully contained.

The probability that a neutrino suffers a charge current interaction is given by

$$
P_{\mathrm{conv}}=n \sigma_{\mathrm{cc}} l
$$

where $n$ is the number density of nucleons of the medium transversed by the neutrino, $\sigma_{\mathrm{cc}}$ is the charged current cross section for a neutrino nucleon interaction and $l$ is the dis- 
tance traveled by the neutrino inside the detector. We determine $\sigma_{\mathrm{cc}}$ according to the CTEQ4 deep inelastic scattering (DIS) distributions, as described in [21]. For energies below $10 \mathrm{GeV}$ a correction on the cross section due to quasi-elastic and resonant effects [22] should be included. As our analysis starts with energies above $10 \mathrm{GeV}$ we do not include these corrections. by

The number of contained events $\left(N_{\text {cont }}\right)$ is therefore given

$$
N_{\mathrm{cont}}=P_{\mathrm{conv}} \varphi_{\oplus} A=n \sigma_{\mathrm{cc}} \varphi_{\oplus} V,
$$

where $\varphi_{\oplus}$ is the flux of upward going neutrinos, $A$ is the detector area and $V$ is the detector volume for contained neutrino interactions.

The neutrino flux determined in the previous section will suffer a minor attenuation when going through the Earth. This attenuation is not significant at these low energies but we will include it for completeness. The differential flux is given by

$$
\frac{d \varphi}{d x}=-n \sigma_{\mathrm{cc}} \varphi
$$

where $\varphi$ is the atmospherical neutrino flux, $x$ is the distance traveled by the neutrino. The atmospheric neutrino flux after transversing the Earth will be given by

$$
\varphi_{\oplus}=\varphi_{0} e^{-\int n \sigma_{\mathrm{cc}} d x} \simeq \varphi_{0} e^{-n_{\oplus} \sigma_{\mathrm{cc}} L} \sim \varphi_{0} e^{-2 n_{\oplus} \sigma_{\mathrm{cc}} R_{\oplus} \cos \theta_{Z}}
$$

where $R_{\oplus}$ is the Earth radius and $\varphi_{0}$ is the initial neutrino flux, the approximations are for constant density and negligible detector depth, respectively.

The volume of the detector available for the neutrino to interact and produce a contained muon depends on the muon range $\left(R_{\mu}\right)$. Making the approximation that the detector has a cylindrical shape, the effective volume $\mathrm{V}$ will be given by

$$
V=\frac{1}{2} h D_{d}^{2} \arcsin \left(\sqrt{1-\frac{R_{\mu}^{2}}{D_{d}^{2}} \sin ^{2} \theta_{Z}}\right)\left(1-\frac{R_{\mu}}{h}\left|\cos \theta_{Z}\right|\right)
$$

where $h$ is the detector height and $D_{d}$ is the detector diameter.

For muons with energies of tens of $\mathrm{GeV}$, the average muon range can be determined analytically by the equation [23]

$$
R_{\mu}=\frac{1}{b} \ln \left(1+\frac{b}{a} E_{0}\right)
$$

where $a$ and $b$ are respectively ionization and radiation loss parameters and $E_{0}$ is the initial muon energy. The average $E_{0}$ is equal to the neutrino energy minus the average energy loss in a charged current interaction. The fractional energy loss is given in [24] and for neutrinos energies between 10-100 $\mathrm{GeV}$ is 0.48 . At these energies $a$ and $b$ can be approximated to constant values where $a=2 \times 10^{-3} \mathrm{GeV} \mathrm{cm}^{2} / \mathrm{g}$ and $b=4$ $\times 10^{-6} \mathrm{~cm}^{2} / \mathrm{g}[23]$.
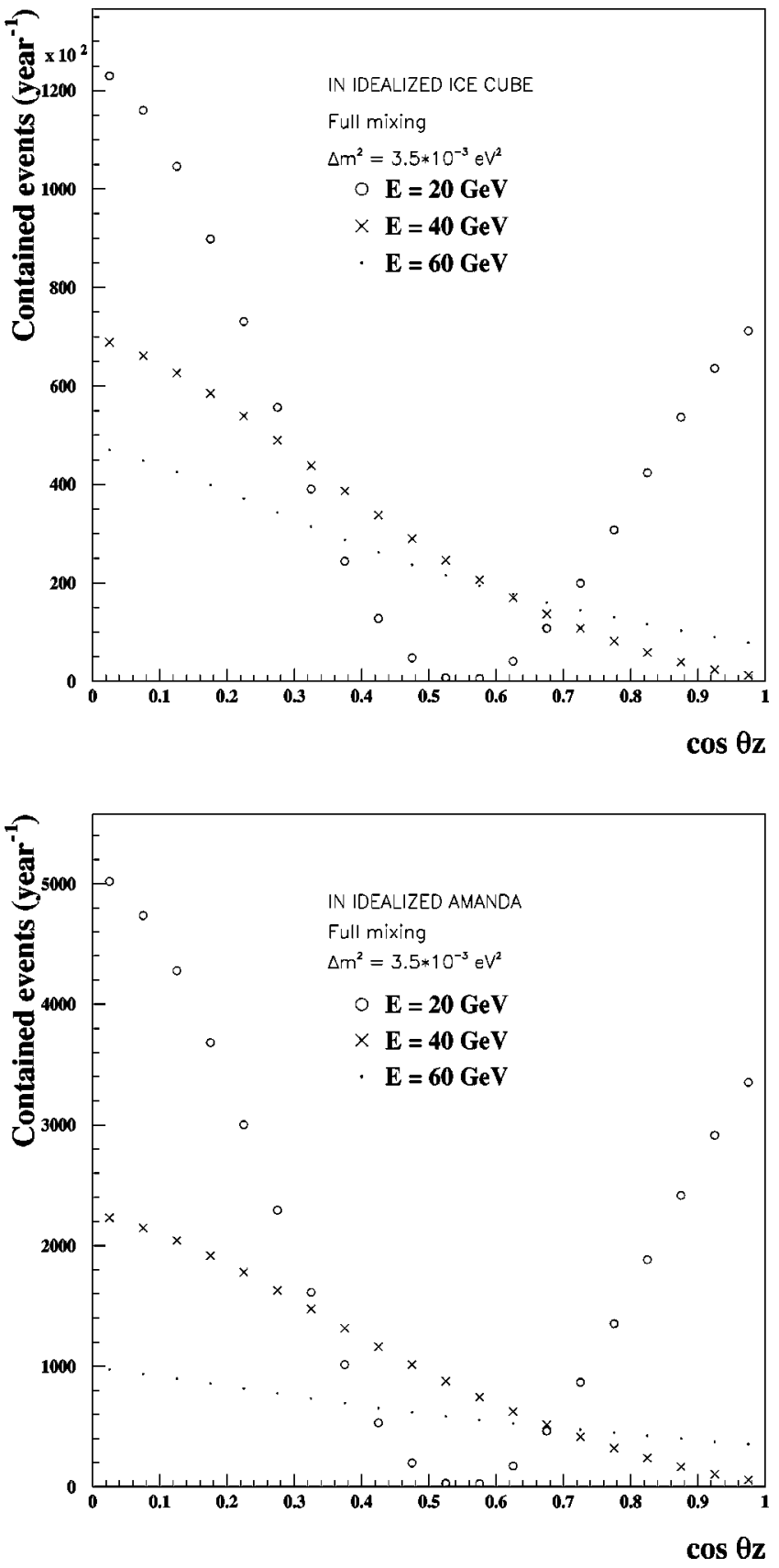

FIG. 8. Annual contained events in idealized IceCube and AMANDA (as labeled) in $0.05 \cos \theta_{Z}$ bins versus cosine of the zenith angle. The neutrino energy is fixed as labeled.

Figure 8 shows the expected number of contained events per year for an idealized detector of the volume proposed for IceCube [15] with height and diameter of a kilometer. The neutrino energy is fixed at 20,40 or $60 \mathrm{GeV}$ and $\cos \theta_{Z}$ divided in bins with a width of 0.05 . Each bin corresponds to about $0.3 \mathrm{sr}$. The same is shown for the idealized detector with the size of AMANDA-II 200-m diameter and 1-km height.

As up to now we are assuming an ideal detector, these figures show that the volume of both AMANDA II and IceCube detectors are quite sufficient to measure atmospheric neutrino oscillations. The key parameter for an ide- 
alized detector is that the energy threshold has to be less than $30 \mathrm{GeV}$. The oscillation pattern for energies below this threshold can be seen when measuring the number of contained events versus the neutrino arrival direction.

However, instrumental effects have to be taken into consideration. Before showing the sensitivity region for the $\Delta m^{2}$ and $\sin ^{2} 2 \theta$ parameters that can be acheived with both AMANDA II and IceCube detectors, we will include these uncertainties in our analysis.

\section{INSTRUMENTAL EFFECTS AND LIMITATIONS}

In this section we consider the degradation of the measurement that results from instrumental effects and limitations. Two major issues are (1) angular and (2) energy reconstruction and resolution, since the physics manifests itself as a function of $L / E$ and we have $L$ as a function of $\cos \theta_{Z}$.

There are two kinds of analysis that can be done. One measures the number of contained events with fixed energies (which is easy to visualize by measuring the number of events versus $\cos \theta_{Z}$ ). The other measures the number of events versus the full $L / E$ spectrum.

To detect an oscillation pattern as a function of $L / E$ requires collecting enough events and determining $L / E$ to sufficient accuracy. From observations of the resulting muons and hadronic shower one estimates the incident neutrino arrival angle $\theta_{Z}$ and energy $E$. The distance $L$ is determined from the angle $\theta$ [see Eq. (2)]. The fractional error in $L / E$ is given by

$$
\frac{\sigma_{L / E_{\nu}}^{2}}{(L / E)^{2}}=\frac{\sigma_{E_{\nu}}^{2}}{E_{\nu}^{2}}+\frac{\sigma_{L}^{2}}{L^{2}} \simeq \frac{\sigma_{E_{\nu}}^{2}}{E_{\nu}^{2}}+\tan ^{2} \theta_{Z} \sigma_{\theta_{Z}^{2}}
$$

where $E_{\nu}$ is the neutrino energy and in the approximation that $L=2 R_{\oplus} \cos \theta_{Z}$ which is reasonably accurate for upward going events. For the next step we assume that the angle and energy correlation is small. This is likely to be only partially correct but will show capabilities.

\section{A. Angular reconstruction and resolution}

The muon does not travel and thus point in the same direction as the incident neutrino except when averaged over many events. This difference occurs because there is some exchange of energy (inelasticity) and momentum to the nucleon when the neutrino converts into a muon. The inelasticity of the interaction and the spread in pointing are related although not as simply as one would like. Figure 9 shows the distribution of the angle $\theta$ between the muon and neutrino directions for several relevant energies.

Characterized by one parameter, the rms spread in direction between the incoming neutrino and the outgoing muon direction is given by the relation [25]

$$
\theta_{\mathrm{rms}} \simeq \sqrt{\frac{m_{p}}{E_{\nu}}}
$$

where the angle is given in radians.

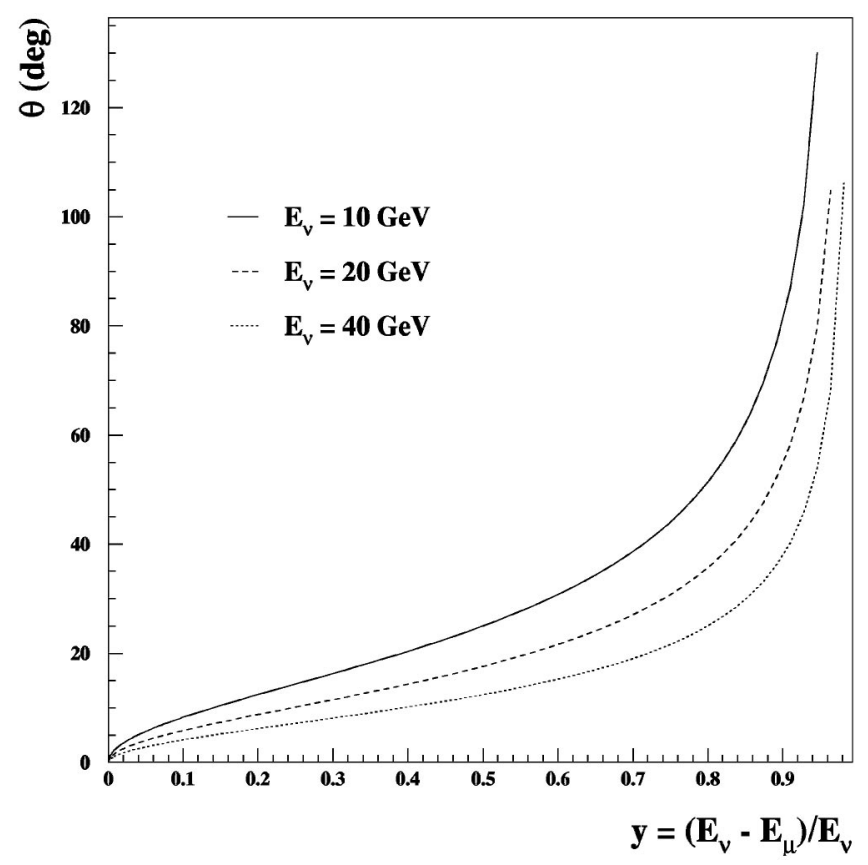

FIG. 9. Angle distribution for several energies (as labeled). $\theta$ corresponds to the angle between the neutrino and the muon directions.

The rms for $\theta$ versus neutrino energy is shown in Fig. 10. This relation is valid for neutrino energies between 10 and $3000 \mathrm{GeV}$. Figure 10 shows that for neutrino energies of a few tens of $\mathrm{GeV}$, the angular rms varies from about 8 to 17 degrees. The rms is spread enough that the direction of the neutrino will only be known by the average of the angular distributions of many events.

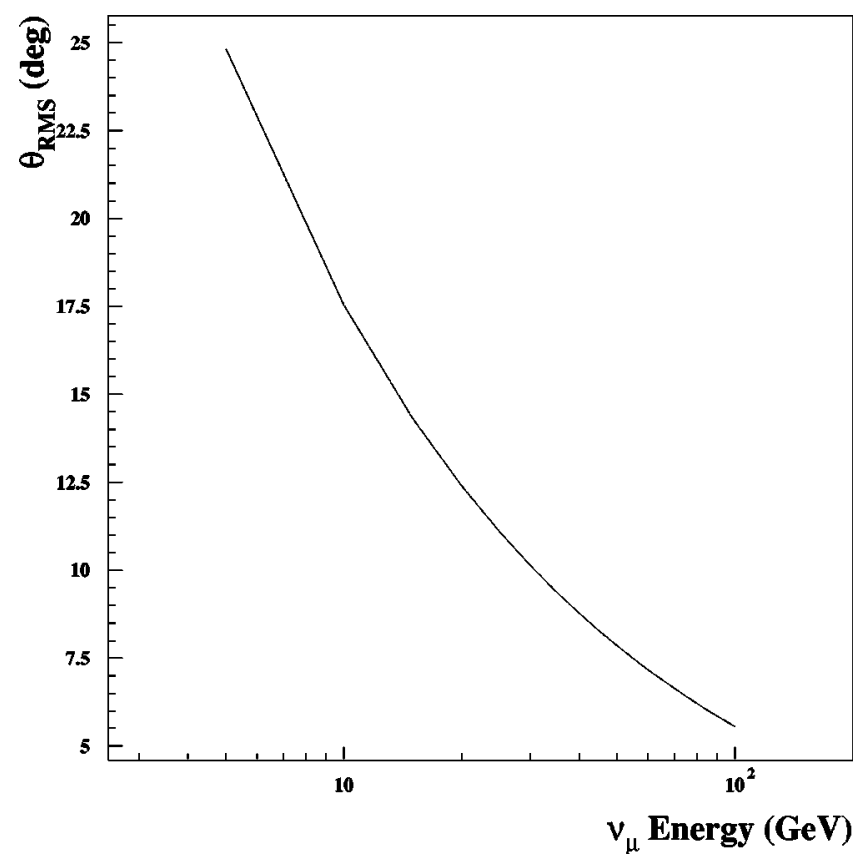

FIG. 10. Angle distribution as a function of neutrino energy. $\theta_{\text {rms }}$ corresponds to the rms angle between the neutrino and the muon directions. 


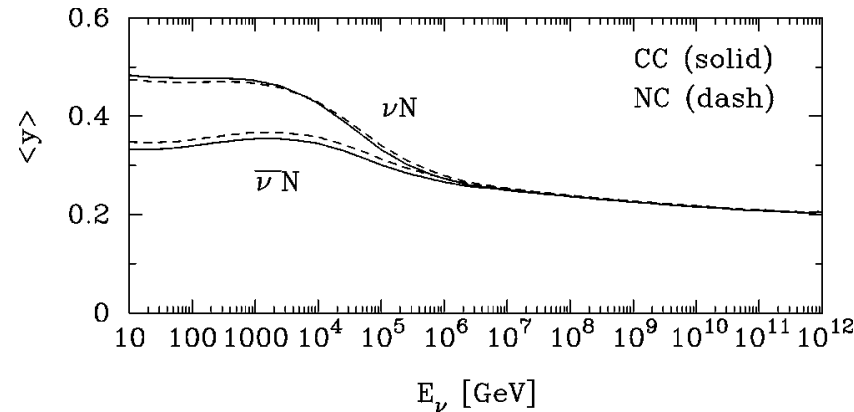

FIG. 11. Mean inelasticity versus energy. $\langle y\rangle=\left(E_{\nu}-E_{\mu}\right) / E_{\nu}$. Figure extracted from [24].

Figure 11 (extracted from [24]) shows the mean inelasticity $\langle y\rangle\left[\right.$ where $\left.y=\left(E_{\nu}-E_{\mu}\right) / E_{\nu}\right]$ versus neutrino energy. In a charged current interaction $\langle y\rangle$ is almost constant between 10 and $100 \mathrm{GeV}$ with a value of 0.48 . Figure 9 shows that this corresponds to an average scattering angle of about $15^{\circ}$ for a $20 \mathrm{GeV}$ neutrino.

The muon direction is determined by measuring the arrival time of the Cherenkov light at each phototube. A muon of energy $E$ will have a mean range of $5 E \mathrm{~m} / \mathrm{GeV}$. If timing is done to $\delta t$, the direction of the muon can be measured to about

$$
\begin{aligned}
\delta \theta=\frac{\sqrt{2} c \delta t}{5 E} \frac{\mathrm{rad} \mathrm{GeV}}{\mathrm{m}} & =\frac{0.085 \delta t}{E} \frac{\mathrm{rad} \mathrm{GeV}}{\mathrm{ns}} \\
& =\frac{4.86^{\circ} \delta t}{E} \frac{\mathrm{GeV}}{\mathrm{ns}}
\end{aligned}
$$

For approximately upward moving muons the direction can also be determined by timing arrival of photons along a vertical string of tubes and comparing it to the speed of light. This technique measures $\cos \theta_{Z}$ directly and with reasonable parameters to an accuracy better than 0.1 .

In the energy range that we are interested in $(10 \leqslant E$ $\leqslant 100 \mathrm{GeV}$ ) the angular resolution is therefore dominated by the neutrino-muon scattering distributions. In our analysis we will consider the average scattering angle. For more precision a Monte Carlo analysis can be done, but we consider the average scattering angle precise enough for the purpose of predicting the possibility of measuring neutrino oscillations. Also, Figs. 2 and 3 show that to observe a $\Delta m^{2}$ effect, observations do not have to be made near the horizon where the path length changes rapidly with angle. Thus the intrinsic spread in path length due to angular error is sufficiently small.

One can therefore expect that for energies above about 10 $\mathrm{GeV}$ the angle of the incoming neutrinos can be determined sufficiently accurately for neutrino oscillation studies.

\section{B. Energy reconstruction and resolution}

We now include uncertainties on the energy estimation of the incident neutrino. An ideal detector, that is equally and fully sensitive to all energy deposited in the detector, could reconstruct the incident neutrino energy very precisely for all fully contained events.

If all the energy from the neutrino went into the muon, then one could exploit the range energy relation and reconstruct the muon and thus the neutrino energy.

We can write the formula for the error in $E_{\nu}$ as

$$
\begin{aligned}
& \sigma_{E_{\nu}}^{2}=\sigma_{E_{\mu}}^{2}+\sigma_{E_{h}}^{2} \\
& \frac{\sigma_{E_{\nu}^{2}}}{E_{\nu}^{2}}=\frac{\sigma_{E_{\mu}}^{2}}{E_{\mu}^{2}}(1-y)^{2}+\frac{\sigma_{E_{h}}^{2}}{E_{h}^{2}} y^{2}
\end{aligned}
$$

where $E_{\nu}, E_{\mu}$, and $E_{h}$ are the neutrino, muon, and hadronic energies, and $y=E_{h} / E_{\nu}$ is the inelasticity of the interaction. Note $E_{\nu}=E_{\mu}+E_{h}$, and $1-y=E_{\mu} / E_{\nu}$.

The muon energy is determined by measuring its rangepath length - which is on average 5 meters per GeV. Thus a determination of the path length to an accuracy of 5 meters corresponds to an error of $1 \mathrm{GeV}$ independent of the energy of the muon. The fluctuations in range have a slight energy dependence.

One has to take the inelasticity in the conversion of neutrino to muons into account. Figure 11 (extracted from [24]) shows that for energies between 10 and $1000 \mathrm{GeV}$ the muon carries about $52 \%$ of the incident neutrino energy. The inelasticity $y$ ranges nearly uniformly for the energy range of interest here. At much higher energies it decreases asymptotically to about $20 \%$ inelasticity.

Thus to reconstruct the neutrino energy the detector must estimate both the muon energy (which is given by the muon range measurement and therefore approximately independent of the energy of the muon) and the inelastic portion. Roughly, the energy resolution is composed of a component which is nearly linearly dependent on energy and a component which is nearly independent of the energy. The muon range is estimated by the observations by optical detectors of the Cherenkov radiation as it travels through the ice (or water). In general, the total light emitted is proportional to the energy deposited. The muon is a line source of light while the other interaction products are generally more localized and near the point of neutrino interaction.

The errors in calibration and fluctuations in the fraction of the energy transferred to the muon and to the other products of the interaction result in an error which is roughly proportional to the energy of the incident neutrino. This uncertainty actually decreases somewhat from very low energies to higher energies.

We can therefore rewrite Eq. (14) as the standard deviation of a function which depends on the neutrino energy: $\sigma_{E}^{2}=x^{2} E^{2}+(\delta E)^{2}$ where $x$ is a constant between 0 and 1 and $\delta E \sim 1 \mathrm{GeV}$, assuming no correlation between these terms. The energy resolution is therefore a fraction of the energy value plus a constant. We give our results assuming 0,10 , and $20 \%$ energy resolution.

The number of contained events in IceCube and in AMANDA-II assuming the above energy resolution are shown in Fig. 12. What one finds is that the standard devia- 

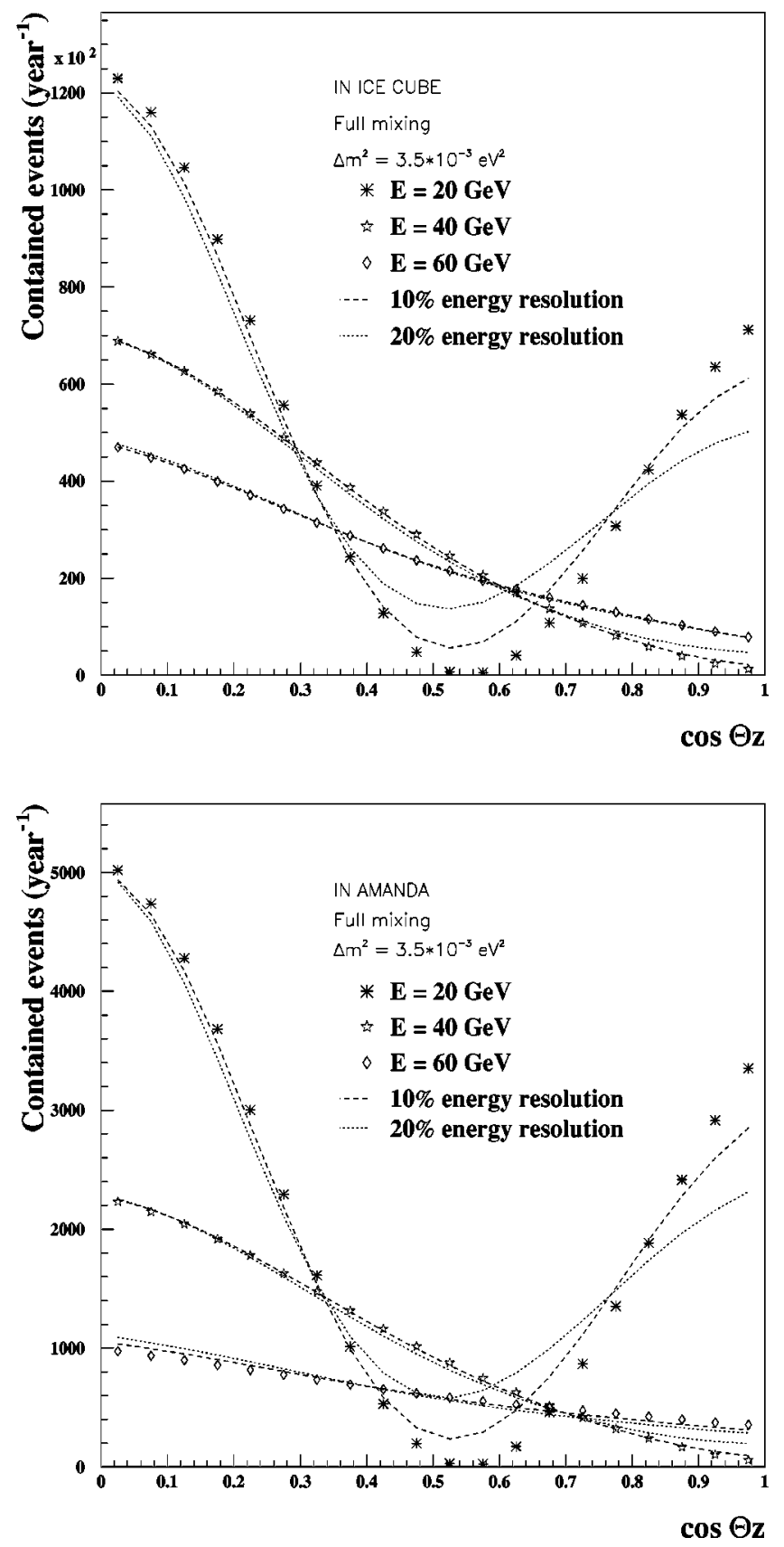

FIG. 12. Contained events in IceCube and AMANDA (as labeled) volume in $0.05 \cos \theta_{Z}$ bins. The neutrino energy is fixed as labeled and the effect of 10 and $20 \%$ energy resolution is shown.

tion must be less than about $20 \%$ of the energy [or full width at half-maximum (FWHM) less than about 50\%] in order to make quality oscillation observations.

The energy threshold is also important. To be able to observe the oscillation pattern the energy threshold can not be much above $20 \mathrm{GeV}$.

Figure 13 shows the survival probability versus $E / L$ (or $E / \cos \theta_{Z}$ ) including 10 and $20 \%$ energy resolution. In this kind of analysis, vertical upwards events $\left(\cos \theta_{Z}=1\right)$ are the best for measuring neutrino oscillations.

IceCube [15] is planned to have 81 strings in a $\mathrm{km}^{3}$ (125 $\mathrm{m}$ between each string). Each string will have 60 photomul-

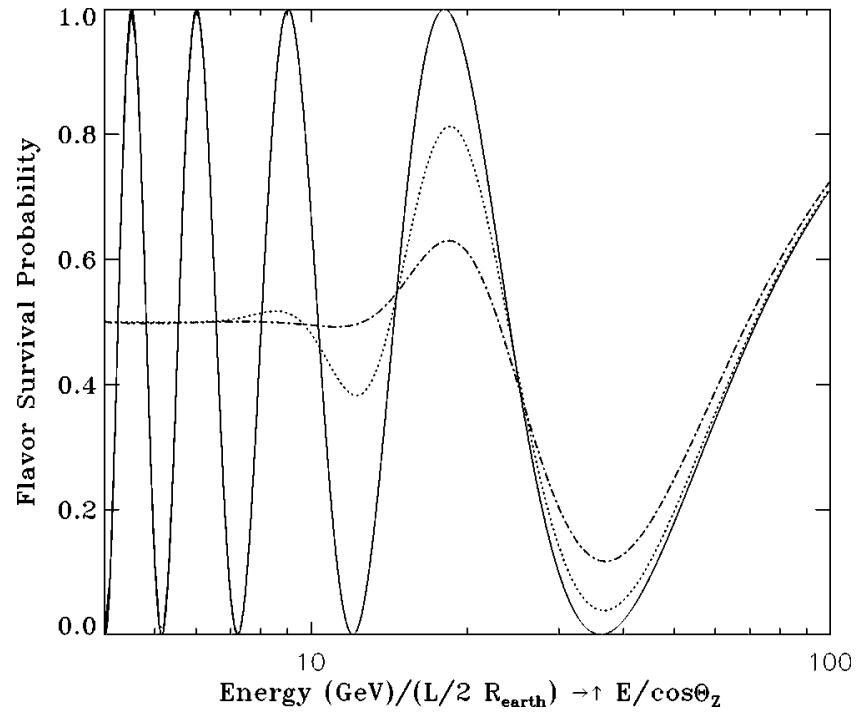

FIG. 13. Flavor survival probability versus $E / \cos \theta_{Z}$. Valid for upwards going neutrinos, where the distance traveled can be given in Earth radius units. The dashed line corresponds to the effect of $10 \%$ and the dotted dashed to $20 \%$ energy resolution.

tiplier tubes (PMTs) with $16 \mathrm{~m}$ between each optical module. AMANDA-II has 4 inner strings with 20 PMTs in each of them; they are located in a cylindrical shape of $60 \mathrm{~m}$ diameter and $400 \mathrm{~m}$ length. There are also two outer set of PMTs; one has 6 strings with 36 PMTs per string and $120 \mathrm{~m}$ diameter (same height). The outermost set has 9 more strings, 700 $\mathrm{m}$ long and instrumented every $14 \mathrm{~m}$ (50 PMTs per string). The diameter of this outer string array is $200 \mathrm{~m}$.

Both AMANDA and IceCube were designed to detect higher energies neutrinos. In both experiments, for atmospheric neutrino oscillation measurements, the strings and phototubes are too far apart to acheive the necessary energy resolution. Also the detector energy threshold is set too high. This is true for a fixed energy analysis (which would require an energy threshold around $20 \mathrm{GeV}$ ) and for the full energy spectrum $(10-100 \mathrm{GeV})$ analysis.

For atmospheric neutrino oscillation measurements the current design proposed for IceCube has two major shortcomings:

(1) Rejection of confusing events is too poor. The individual strings of optical modules are too far apart to guard neighboring strings against muons coming near a string and appearing to be a contained event or multiple muons depositing energy or light near a string and simulating a contained event. The rate of downward muons and muon bundles is about a million times the contained event rate.

(2) The vertical optical module spacing is also too great (by a small factor) to provide sufficient energy resolution even for the vertical going contained events. One can estimate that with $16-\mathrm{m}$ spacing that the range of the muon can be measured to about 8 to 10 meters rms giving an error of about $2 \mathrm{GeV}$ rms for the muon energy. However about half the energy of the original neutrino energy is deposited in the hadron shower but in a very wide distribution which ranges basically from 0 to $100 \%$. Thus it is necessary to determine how much energy is deposited inelastically, which can be 
done by measuring how much light comes from the hadronic or electronic shower to the optical module and then estimating how much energy was deposited by determining how far it was from the shower to the optical modules. With a single string the vertical distance of the main energy deposition can be determined, though somewhat too poorly, but the distance away from the string is much more poorly constrained. For example, if the distance to the inelastic energy deposition is determined to 2 to 3 meters out of a typical distance of 10 meters, then the error in estimating the distance translates into an error of $40 \%$ or more in reconstructing the inelastic energy. There is also an intrinsic spread in the amount of light received due to the statistical fluctuations in transit to 16-m separated optical modules. The net result is that the total energy resolution for vertical going contained events is too poor by a significant amount and is worse for other angles.

The current version of AMANDA-II also lacks the resolution necessary for atmospheric neutrino oscillation measurements. At the present the trigger system and experimental procedures are set for measuring higher energy neutrinos with a high energy threshold (about $50 \mathrm{GeV}$ ) and discriminate against contained events.

AMANDA-II is closer to the necessary density of strings and optical modules than IceCube, but the vertical spacings of 20 meters, 11 meters, and 14 meters and average horizontal spacing of 40 meters is just too large to have the required energy resolution. The set of six strings with 11-meter vertical spacing would be nearly adequate but need additional infrastructure in terms of other strings nearby, calibration of the optical module response and the appropriate triggering and data processing software. Based upon the optical properties of the ice and optical modules in situ and the performance of the detectors, we estimate that a spacing of order 5 to 10 meters would be adequate.

Similar arguments hold when it comes to utilizing AMANDA-II and IceCube for future long baseline neutrino oscillation experiments [27]. If the beams originate from CERN or Fermilab, latitudes about $46^{\circ}$ and $43^{\circ}$ north respectively, then a typical angle of the incoming neutrino and produced muon is about $45^{\circ}$ to the zenith and there is a noticeable spread in the muon directions. For that range of angles, the optical horizontal separation is as important as the vertical separation. (Also note that the rough $45^{\circ}$ zenith angle makes the effective vertical separation $\sqrt{2}$ or $41 \%$ greater.) Though long-baseline experiments have the advantage of timing to reduce backgrounds and thus enable lower energy thresholds set by the experimental trigger [27], the angles for likely beams lowers the energy resolution substantially. The neutrino beam effective volume for IceCube would be quite low due to the large spacing between strings.

\section{TOWER DETECTOR CONFIGURATION}

It is clear that measuring various values of $L$ and $E$ provides a very good and self-consistent test of neutrino oscillations. However, it is possible to test and observe neutrino oscillations for a fixed distance, providing sufficient range of energy and number of neutrinos can be observed.

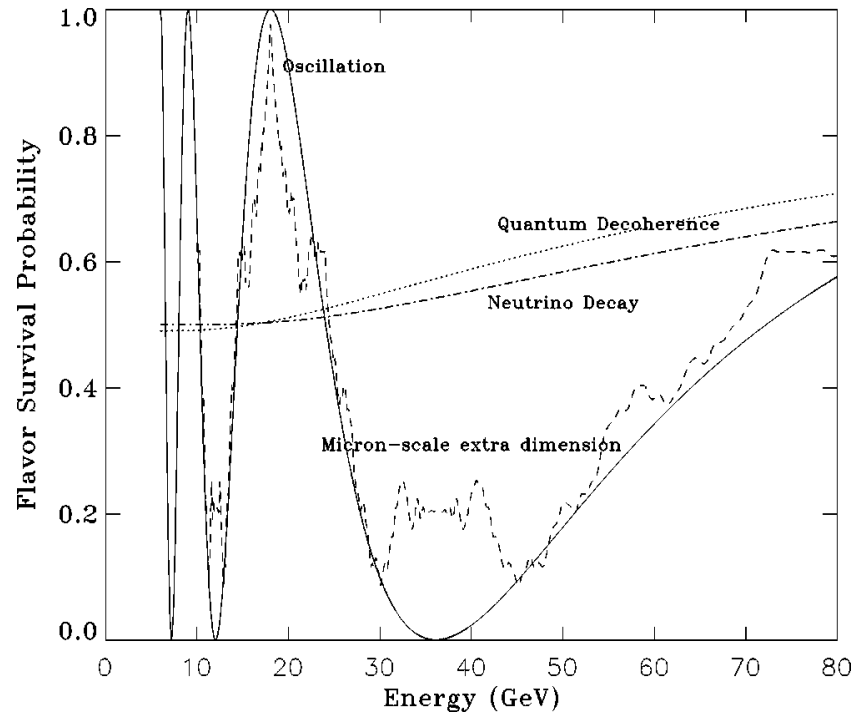

FIG. 14. Muon neutrino survival probability versus neutrino energy for upwards going neutrinos $\left(L=2 R_{\oplus}\right)$. Again four theoretical scenarios that might account for the observed effect are: (1) Standard neutrino oscillations are shown by the solid line. Full neutrino mixing and $\Delta m^{2}=3.5 \times 10^{-3} \mathrm{eV}^{2}$ is assumed. (2) Dashed line is effect of additional dimension with characteristic radius of about a micron [8]. (3) Dashed dotted line is effect of a decaying neutrino [7]. (4) Dashed line is the effect of quantum gravity decoherence $[9]$.

Figure 14 shows the muon survival probability versus energy $E$ for upwards going neutrinos $\left(L=2 R_{\oplus}\right)$ superimposed by non-standard explanations.

We now consider explicitly a configuration in the shape of a long tower. This tower is oriented vertically both to get the maximum path length $L \simeq 2 R_{\oplus}$ and because that is operationally the most reasonable configuration for water and icebased detectors. The tower geometry has its height much longer than its diameter so that its acceptance is near vertical (near direct upward and downward going directions) and its solid angle is quite limited. The limited solid angle means that the distance is effectively constant (due to the slow change in $\cos \theta$ near 0 and 180 degrees). Thus the blurring due to the change in $L$ adds very little to the blurring caused by the energy resolution.

We consider the hypothetical configuration with optical detectors at $5-\mathrm{m}$ to $10-\mathrm{m}$ spacings along a string $1-\mathrm{km}$ long. The close optical detector spacing improves the energy resolution both in terms of determining the muon range and in the energy deposited by the other interaction products. The tower detector consists of four such strings embedded in a larger detector, e.g., AMANDA-II. The larger detector acts as an after the fact additional veto and, when appropriate, provides additional information in constraining the event and its energy.

Figure 15 shows the number of contained events per year versus energy for vertical going neutrinos. In order to get the atmospheric muon neutrino flux at lower energies, we extrapolate the Volkova [18] spectrum used in Sec. III to 5 $\mathrm{GeV}$. We compared this flux with the one calculated in [20] and they are in good agreement. 


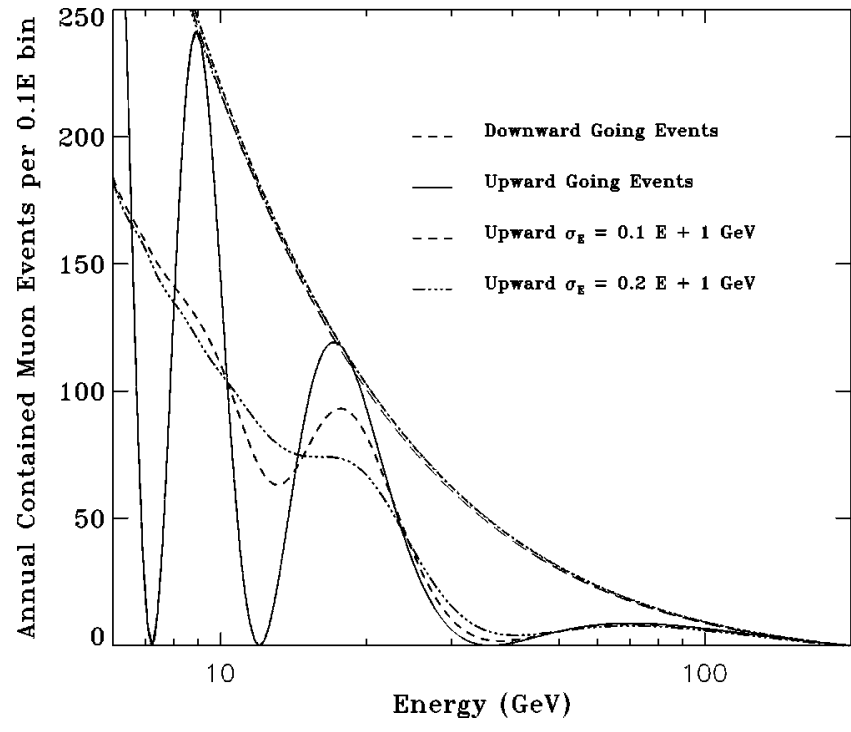

FIG. 15. Annual contained events for tower configuration including a 10 and $20 \%$ energy resolution.

This figure shows the expected event rates as a function of energy with perfect energy resolution and including a 10 and $20 \%$ energy resolution effect.

Previous figures in this section assume the measurement of the neutrino energy through measuring the muon energy and hadronic energy. Another approach to observing neutrino oscillations is through the measurement of the muon range, without the information on the hadron energy and reconstruction of the neutrino energy. Such a measurement would have the advantage of a clean estimate of muon energy. Since the muon carries a variable fraction of the energy of its parent neutrino, this is equivalent to having a random error in the neutrino energy estimate with a bias to the low energy side. This bias is not overwhelming because the atmospheric neutrino energy spectrum is steeply falling and the number of muons observed at energy $E_{\mu}$ is dominated by parent neutrino energies which are just above the muon energies rather than those from much higher energies.

Figure 16 shows the ratio of the number of events with oscillations over the number of events without oscillations versus the neutrino energy and versus the muon energy. Measurement of neutrino oscillations using the muon energy information alone is still possible. This kind of analysis is proposed for ANTARES [26] and their predicted response is shown in Fig. 21. Figure 16 shows that our proposed tower configuration can measure neutrino oscillations in the same way, that is, using only the muon energy information. The key issue in that approach is to determine the ratio of upward going versus downward going contained muon events.

\section{BACKGROUNDS AND SYSTEMATICS}

This paper is not meant to be a complete analysis of the Tower configuration for neutrino oscillation observations. More work is necessary. However, it is appropriate to outline and scope anticipated backgrounds and systematic errors to see both if there are any potential show stoppers and which areas need further work.

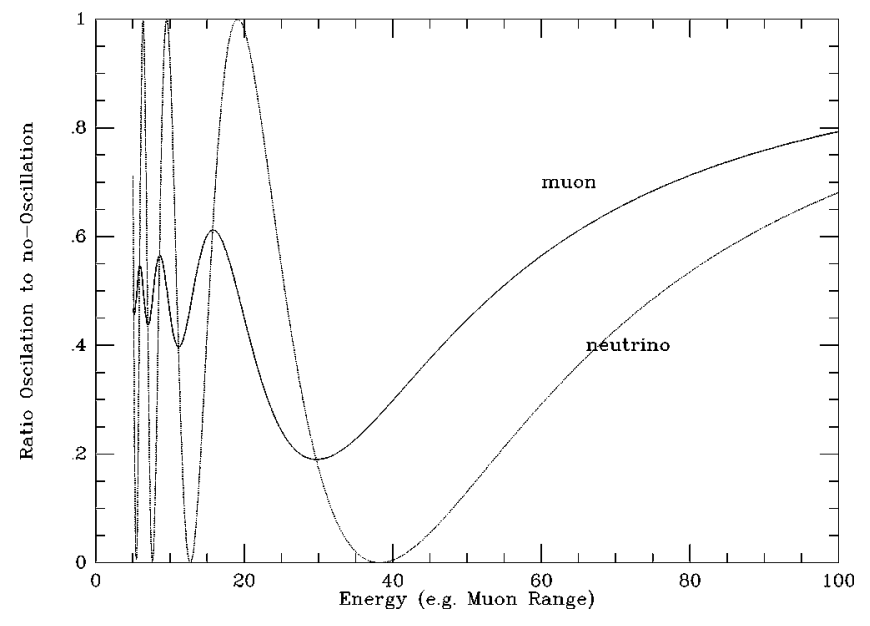

FIG. 16. Ratio of the number of events with oscillations over the number of events without oscillations versus the neutrino energy and versus the muon energy $(\mathrm{GeV})$.

\section{A. Atmospheric muons}

The primary background is due to the very numerous downgoing muons produced by cosmic rays hitting the atmosphere. This is the same process that produces the atmospheric neutrinos.

A veto against these events puts a more stringent requirement than that needed to ensure that an event is contained. A special trigger is required that looks for events and event topology inside the active area of the detector and vetoes against particle entering the detector volume from above and below or from the side. An AMANDA style low multiplicity majority logic trigger would be dominated by random coincidence from optical module noise. Deadtime due to the veto is an issue so that separate triggers would be necessary for separate science goals. One must also be concerned about self-vetoing due to photons leaking from the real signal because the absorption length in ice is fairly long. Overcoming this kind of self-vetoing requires careful timing which may in turn require after the fact processing of a much larger data set.

The observation must have very good rejection of muons coming in at an angle and thus appearing to be a contained event with very little inelasticity. This is the primary motivation for locating the denser optical module strings inside the existing AMANDA-II array. The combination of the Tower closed-packed and dense strings and the information from the 19 AMANDA-II strings provides good rejection of well off-vertical muons.

\section{B. Electron neutrinos}

At low energies (order of $10 \mathrm{GeV}$ ), electron neutrino charged-current interactions could mimic low energy muon neutrino induced events. The fluxes are similar. Some are likely to be included in the data sample and a careful study would be needed to show that they can readily be rejected to the necessary level. Fortunately, one can test the results using only the higher energy events at some cost in sensitivity. 


\section{Neutral current interactions}

Although Super-Kamiokande favors muon neutrino oscillation into tau neutrino [13], there is still the possibility that it oscillates into sterile neutrinos. If the latter is the case, muon neutrino neutral-current interactions are also a background to be considered. They will oscillate as the signal and due to an inelasticity comparable to the charged current one (see Fig. 11) will produce a jet of events from the nucleon recoil. This generates some oscillation in the energy spectrum of this background. The difficulty in distinguishing neutral-current and charge-current events is that the muon range and the photon range in ice are similar. So photons produced in the neutral current jet can reach the same optical modules as the muon Cherenkov light, and with fluctuations being what they are, it is easy to misreconstruct the vertex of the interaction and/or muon range.

Vertex reconstruction is very difficult for short range (50 $\mathrm{m})$ muons leaving the neutrino interaction vertex. Without good vertex, the energy resolution degrades. To get energy resolution of order $20 \%$ requires that the vertex is very well known. This requires careful timing and calibration of the optical modules.

This background can be understood as long as the detection and reconstruction efficiencies are the same as for the charged current events. Count rates will be lower with realistic reconstruction efficiencies, which at these energies is limited to some extent by the muon neutral current interaction background.

\section{Tau neutrinos}

If there are neutrino oscillations, as other explanations (as shown in Fig. 14), the muon neutrino, most likely they oscillate into tau neutrinos [13]. If this interpretation is correct, the tau neutrinos present a background which tends to wash out the oscillations.

The outgoing tau from the tau neutrino charged current interactions decays to muons about $18 \%$ of the time. Thus the secondary muon will look like a muon neutrino charged current event and attenuate the oscillation pattern. This represents a slight decrease in sensitivity and is comparable in effect to an energy degradation.

Another $11 \%$ of taus decays into a pion plus a tau neutrino, and the pion may decay to a muon or occasionally mimic a muon. Another $25 \%$ of the taus decays in to a pion, pi-zero, and tau neutrino, and the pion decays into a muon. At $20 \mathrm{GeV}$, most pions interact before they decay. To do a full evaluation of these effects requires a simulation. The same may be required to determine what fraction of these taus will generate cascade backgrounds.

The tau neutrino-interaction charged-current cross section has a threshold around $3 \mathrm{GeV}$, so any tau background is quite energy dependent and may cease to be important below 10 $\mathrm{GeV}$. This background could be quite noticeable degradation of the signal for this experiment and for several of the others discussed here.

\section{SENSITIVITY TO PARAMETERS AND COMPARISON WITH OTHER EXPERIMENTS}

Figure 17 shows the parameters $\sin ^{2} 2 \theta$ and $\Delta m^{2}$ sensitivity region for the tower configuration described above. This

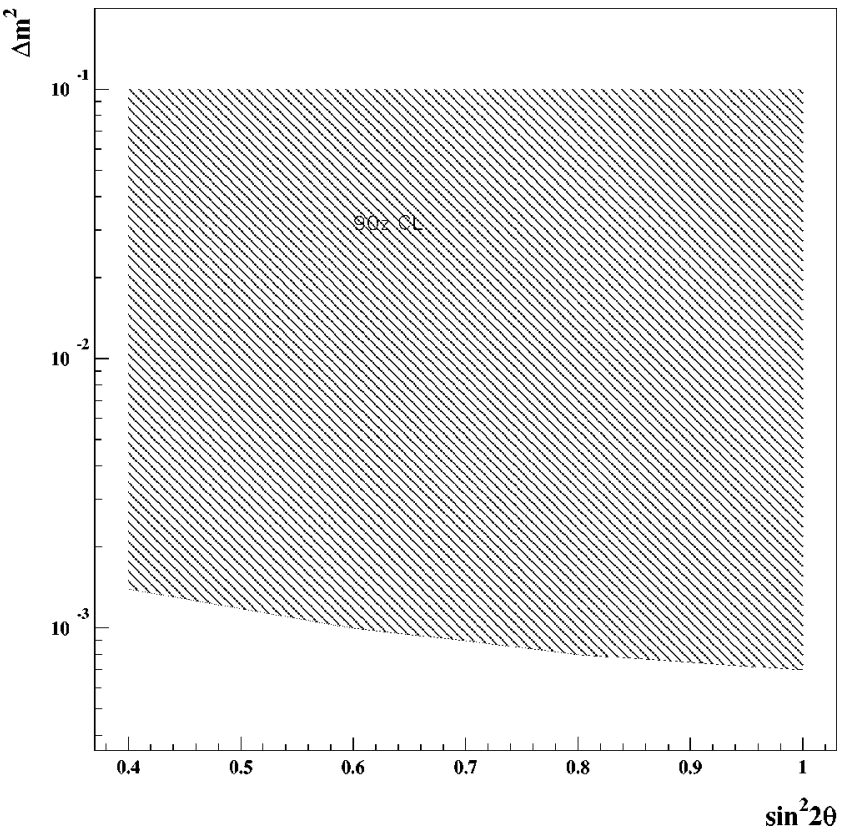

FIG. 17. $\sin ^{2} 2 \theta$ versus $\Delta m^{2}$ sensitive region to demonstrate neutrino oscillation with the proposed tower configuration. The shaded region can be probed with $90 \%$ C.L. and with a precision of $10 \%$ or better in both $\sin ^{2} 2 \theta$ and $\Delta m^{2}$. A detector energy threshold of 15 $\mathrm{GeV}$ and a $10 \%$ energy resolution is assumed.

is the region where one can demonstrate oscillation with $90 \%$ C.L. and a precision of $10 \%$ or better in both $\sin ^{2} 2 \theta$ and $\Delta m^{2}$. We assume that the detector energy threshold is 15 $\mathrm{GeV}$ and the energy resolution is $10 \%$. The sensitivity region for the tower configuration is $\sin ^{2} 2 \theta>0.40$ and $\Delta m^{2}$ $>1 \times 10^{-3}$.

A better precision can be achieved around the most probable values of $\sin ^{2} 2 \theta$ and $\Delta m^{2}\left(\sin ^{2} 2 \theta=1\right.$ and $\Delta m^{2}$ $=3.5 \times 10^{-3}$ ). This is shown in Figs. 18 and 19 for different detector energy threshold and energy resolution. In this region the AMANDA II-Tower configuration expects to have a relative error on $\sin ^{2} 2 \theta$ of order $3 \%$ assuming the projected detector energy threshold of $15 \mathrm{GeV}$ and $90 \%$ C.L. The relative error on $\Delta m^{2}$ is expected to be around $2 \%$ for the same energy threshold and confidence level.

The sensitivity region and the relative error of the parameters were determined by applying a maximum likelihood method [23] assuming a Gaussian distributed data set and using Fisher matrix coefficients. This analysis does not include the effect of systematic errors nor correlates the parameters $\sin ^{2} 2 \theta$ and $\Delta m^{2}$. However, this is not a problem when comparing our proposed configuration with other detectors, since none of them include systematic bias in their sensitivity analysis.

A comparison with other experiments is shown in Table I. MINOS [30] and MONOLITH [31] cover the broadest parameter region. All experiments including our proposed tower configuration have roughly the same sensitivity.

An important point is that the experiments that compose Table I represent three different techniques to measure neutrino oscillations. K2K [29] and MINOS [30] use a controlled beam line and have two detectors, one close to the 


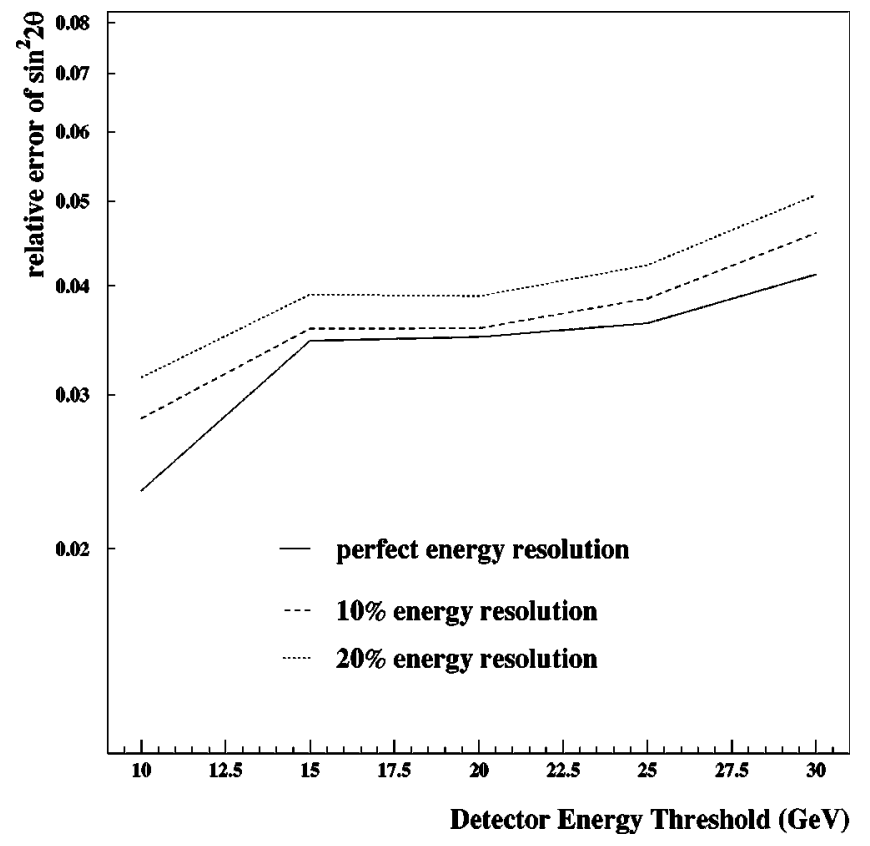

FIG. 18. 90\% C.L. estimated error in the mixing parameter $\sin ^{2} 2 \theta$ versus detector energy threshold. This precision can be acheived around the most probable value for neutrino oscillation $\left(\sin ^{2} 2 \theta=1\right)$ assuming the most probable value for $\Delta m^{2}\left(\Delta m^{2}\right.$ $\left.=3.5 \times 10^{-3}\right)$.

beam production and another one far away. Although the detectors in each experiment are different from each other, the fact that they can control the beam and therefore have a good energy and angular resolution is a strong characteristic of their design.

MONOLITH [31] is a massive and dense magnetized tracking calorimeter. The size and density of the detector are important to increase the number of neutrino interactions and the number of contained events. MONOLITH will cover a large range in $\Delta m^{2}$ since it is able to measure high momentum muons which in other experiments is not possible since the muons range out of the detector before losing most of their energy.

The tower embedded in AMANDA-II that is proposed here and ANTARES are neutrino telescopes with large volumes in water or ice. Their advantage is their large size (detector volume). Both track and measure the energy of the muons from the Cherenkov light emitted in their passage through water or ice.

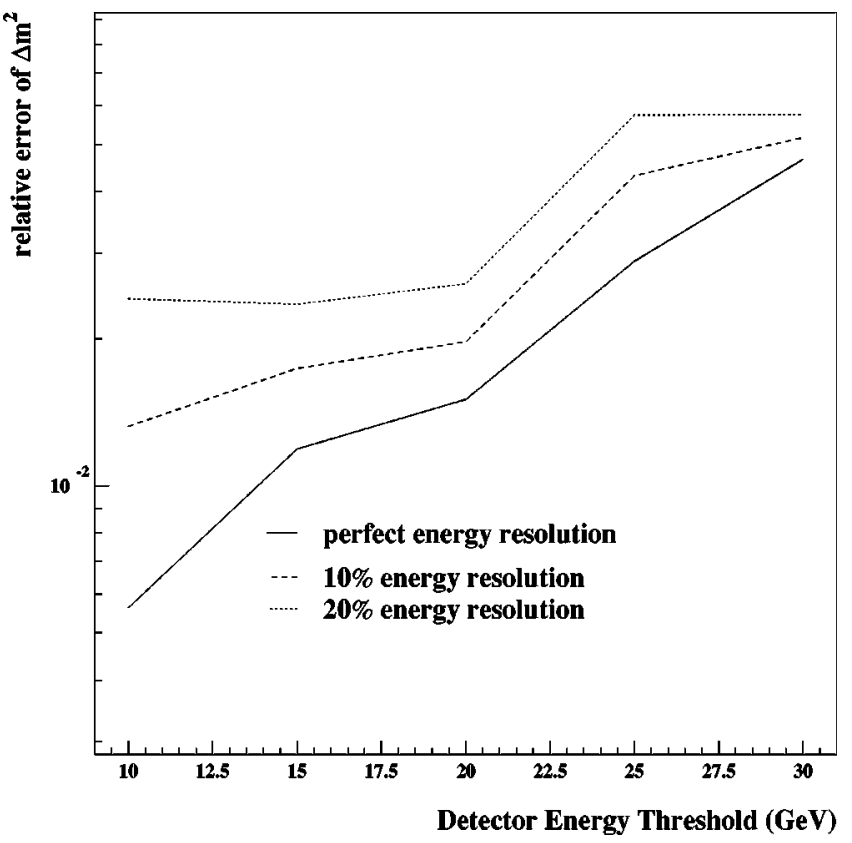

FIG. 19. $90 \%$ C.L. estimated error in the difference in the two neutrino mass squared $\Delta m^{2}$ versus detector energy threshold for the proposed tower configuration. This precision can be achieved around the most probable value for neutrino oscillation $\left(\Delta m^{2}\right.$ $=3.5 \times 10^{-3}$ ) assuming the most probable value for $\sin ^{2} 2 \theta$ $\left(\sin ^{2} 2 \theta=1\right)$.

The current neutrino telescopes as AMANDA, Baikal and future telescopes as IceCube, NESTOR and NEMO have been aimed at detecting astrophysical neutrinos and not designed specifically for neutrino oscillations.

ANTARES, in contrast, has been designed to be sensitive to neutrino oscillation measurements. ANTARES [16] has a smaller net volume than IceCube but has the potential to measure neutrino oscillations in the range of parameters indicated by Super-Kamionkande.

The fact that the neutrino oscillation hypothesis will be tested with different techniques only enhances the importance of these experiments. All experiments will have a good sensitivity around the most probable values found by $\mathrm{Su}-$ perKamiokande [1].

Two other experimental possibilities are UNO [32] and a long baseline experiment that would have a beam coming from either Fermilab or CERN CNGS to IceCube [27]. UNO would be a Cherenkov detector with 20 times the volume of

TABLE I. Comparison among current or proposed neutrino experiments.

\begin{tabular}{lcccccc}
\hline \hline Experiment & $\begin{array}{c}\text { Energy } \\
\text { threshold } \\
(\mathrm{GeV})\end{array}$ & $\Delta m^{2}\left(\mathrm{eV}^{2}\right)$ & $\sin ^{2} 2 \theta$ & $\begin{array}{c}\text { Estimated } \\
\text { precision } \\
\left(\Delta m^{2}\right)\end{array}$ & $\begin{array}{c}\text { Estimated } \\
\text { precision } \\
\left(\sin ^{2} 2 \theta\right)\end{array}$ & C.L.(\%) \\
\hline Proposed Tower & 16 & $>10^{-3}$ & $>0.4$ & $10 \%$ & $10 \%$ & 90 \\
K2K [29] & 1 & $>3 \times 10^{-3}$ & $>0.4$ & $30 \%$ & $?$ & 90 \\
MINOS [30] & 1 & $>10^{-3}$ & $>0.1$ & $10 \%$ & $10 \%$ & 90 \\
MONOLITH [31] & 1.5 & $>2 \times 10^{-4}$ & $>0.2$ & $6 \%$ & $?$ & 90 \\
ANTARES [28] & 5 & $>10^{-3}$ & $>0.6$ & $33 \%$ & $33 \%$ & 99 \\
\hline \hline
\end{tabular}


TABLE II. Time scale for current or proposed neutrino experiments.

\begin{tabular}{lcc}
\hline \hline Experiment & $\begin{array}{c}\text { Start } \\
\text { data taking }\end{array}$ & $\begin{array}{c}\text { Time scale to achieve } \\
\text { expected results } \\
\text { (years) }\end{array}$ \\
\hline Proposed Tower & 2003 & 1 \\
K2K [29] & June 1999 & 3 \\
MINOS [30] & 2003 & 2 \\
MONOLITH [31] & $2005-2006$ & 4 \\
ANTARES [28] & 2003 & 3 \\
\hline \hline
\end{tabular}

SuperKamiokande. It would identify electron and muon neutrino interactions and would have good energy and vertex resolution as well as good reconstruction efficiency (comparable to SuperKamiokande). UNO could do even longer baseline physics to look for $C P$ violation. However this would be in a much longer time scale and UNO would have to adjust its goals according to new results obtained.

The long baseline [27] that proposes a beamline from Fermilab or CERN to IceCube would have to take into account the fact that the angle which the beam would reach the detector is not favorable as described in Sec. V B. Significant modifications would be necessary to the current design of IceCube to make the effort of sending a beam to IceCube work.

Table II shows the quoted time scale for each experiment. $\mathrm{K} 2 \mathrm{~K}$ is the only one already in data taking mode. All others will take two more years to start collecting data.

MINOS will be able to cover a broad $\sin ^{2} 2 \theta$ and $\Delta m^{2}$ parameter space in a reasonable amount of time. Figure 4 of [30] shows their precision in these parameters assuming $\sin ^{2} 2 \theta=0.7$ and $\Delta m^{2}=5 \times 10^{-3} \mathrm{eV}^{2}$. In the same figure they show simulated results where, assuming the above values, they can distinguish among nonoscillations and oscillations. The energy region shown in this figure ranges from zero to $20 \mathrm{GeV}$. The region which probes the oscillation pattern ranges from zero to less than $10 \mathrm{GeV}$.

Figure 15 shows that the Tower configuration can probe the region above $15 \mathrm{GeV}$ and determine the existence or not of the oscillation pattern. Figure 14 shows that the region which can probe the oscillation hypothesis versus nonstandard scenarios that can account for the neutrino deficit, is above about $10 \mathrm{GeV}$. So although MINOS can probe very well the current parameters of neutrino oscillation, the tower configuration will be able to distinguish among different scenarios that can account for the neutrino deficit. Even in the case that the oscillation hypothesis holds, the Tower configuration and MINOS will complement each other results since they are probing different energy ranges in about the same time scale.

Although MONOLITH can also probe a broad parameter space region, it can be considered as another generation of neutrino experiments given their time scale for data taking. Figure 20 (extracted from their proposal [31]) shows that they can also probe the existence of an oscillation pattern well above $10 \mathrm{GeV}$. In this way they will also be able to distinguish among different models which can account for

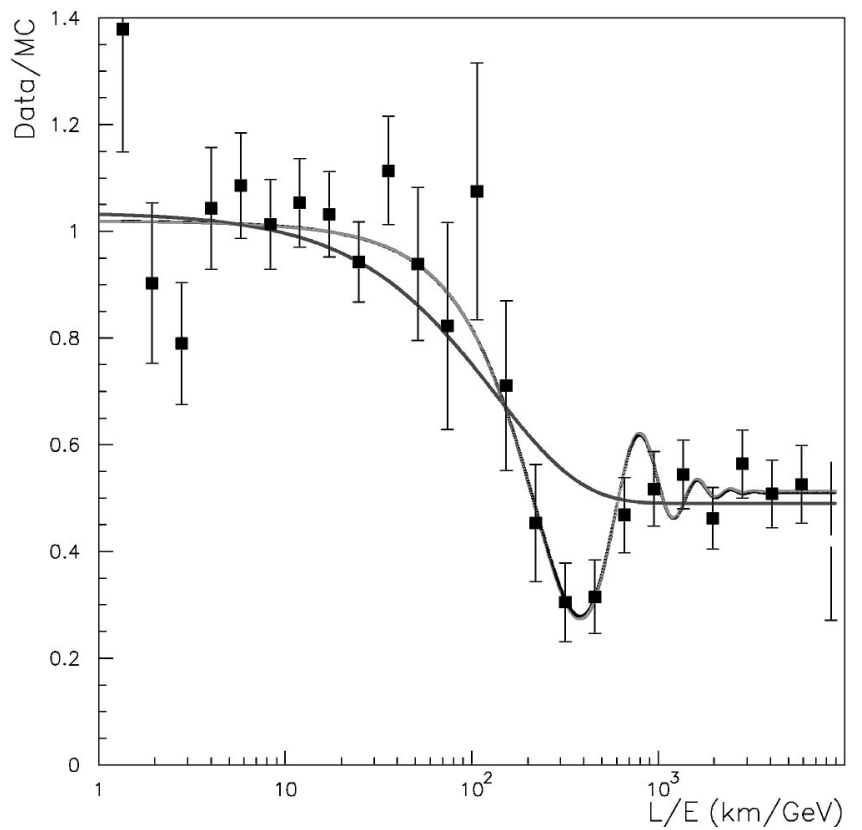

FIG. 20. L/E distribution expected from MONOLITH for $\Delta m^{2}$ $=3 \times 10^{-3} \mathrm{eV}^{2}$ compared to the best fit oscillation hypothesis (oscillating line) and to the best fit of the neutrino decay model (extracted from MONOLITH proposal [31]).

the SuperKamiokande result. However their time scale is far beyond MINOS, K2K and the Tower configuration.

$\mathrm{K} 2 \mathrm{~K}$ also has good sensitivity for the oscillation parameter space and its time scale is comparable with both MINOS and the tower configuration.

ANTARES will also be able to test the oscillation hypoth-

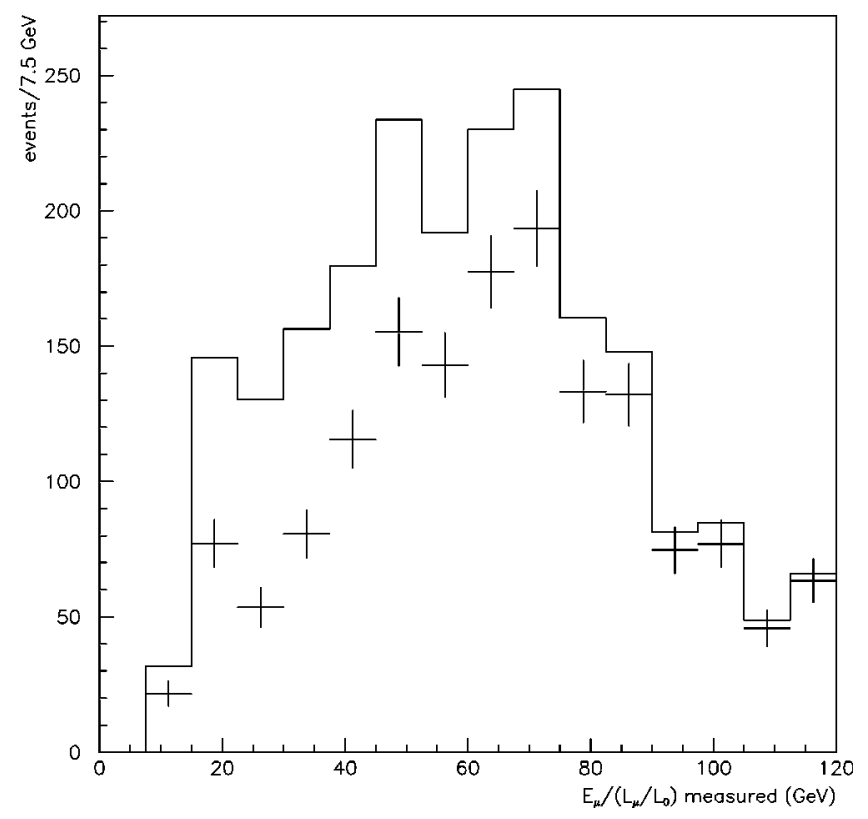

FIG. 21. ANTARES estimated number of reconstructed events versus $E / L$ from muon neutrinos undergoing oscillations with the parameters $\sin ^{2} \theta=1$ (maximal coupling) and $\Delta m^{2}=0.0035 \mathrm{eV}^{2}$ (points) and with no oscillations (histogram) for three years of observations. (Extracted from Antares proposal [26].) 
esis, since they will be able to go to higher energies. Figure 21 shows the potential anticipated observations for 3 years by ANTARES [28]. Their time scale is longer than the Tower configuration since they will need 3 years of data taking. Also the plot of their predicted results is based on the assumption that the atmospheric neutrino angular resolution can be measured to about 3 degrees. We estimate that the intrinsic spread between the observed muon and incoming neutrino is of order 10 degrees.

The comparison made above shows that AMANDA-II modified to include the tower configuration has a strong case for probing the neutrino oscillation hypothesis. Its sensitivity can assure a good constraint in the current allowed region for neutrino oscillations. If the suggested modifications are implemented, the AMANDA detector can demonstrate or rule out atmospheric neutrino oscillations and might point to the correct solution for the neutrino disappearance phenomena.

One could argue that low energy physics should be done with lower energy detectors and this is not the strong suit of IceCube or AMANDA due to the photon scattering and low phototube density. The argument here is that with some increase in phototube density then the Cherenkov water detec- tors can be very effective for atmospheric neutrino oscillation measurements because they can bring large volumes to bear.

\section{SUMMARY}

High energy neutrino telescopes may be adapted to provide very powerful observations of neutrino oscillations and/or some of the alternatives that might explain the up/ down neutrino asymmetry observed by Super-Kamionkande and other anomalies.

\section{ACKNOWLEDGMENTS}

This work supported by NSF Grants KDI 9872979 and Physics/Polar Programs 0071886 and in part by the Director, Office of Energy Research, Office of High Energy and Nuclear Physics, Division of High Energy Physics of the U.S. Department of Energy under Contract No. DE-AC0376SF00098 through the Lawrence Berkeley National Laboratory. We thank Steve Barwick, Wick Haxton, Willi Chinowsky, John Jacobsen, Hitoshi Murayama, and Howard Matis for comments.
[1] Y. Fukuda et al., Phys. Rev. Lett. 81, 1562 (1998).

[2] K.S. Hirata et al., Phys. Lett. B 205, 416 (1988); 280, 146 (1992); Y. Fukuda et al., ibid. 335, 237 (1994).

[3] D. Casper et al., Phys. Rev. Lett. 66, 2561 (1991); R. BeckerSzendy et al., Phys. Rev. D 46, 3720 (1992).

[4] W.M. Allison et al., Phys. Lett. B 391, 491 (1997).

[5] J.N. Bahcall, P.I. Krastev, and A. Yu Smirnov, Phys. Rev. D 60, 093001 (1999).

[6] V. Barger et al., Phys. Rev. Lett. 82, 2640 (1999).

[7] G. Foglie et al., Phys. Rev. D 57, 5893 (1998).

[8] R. Barbieri, P. Creminelli, and A. Strumia, Nucl. Phys. B585, 28 (2000).

[9] E. Lisi, A. Marrone, and D. Montanino, Phys. Rev. Lett. 85, 1166 (2000).

[10] Ch. Berger et al., Phys. Lett. B 245, 305 (1990).

[11] M. Aglietta et al., Europhys. Lett. 8, 611 (1989).

[12] P. Fisher, B. Kayser, and K.S. Macfarland, Annu. Rev. Nucl. Part. Sci. 49, 481 (1999).

[13] S. Fukuda et al., Phys. Rev. Lett. 85, 3999 (2000).

[14] E. Andres et al., Astropart. Phys. 13, 1 (2000).

[15] See the proposal for ICE CUBE at http:// pheno.physics.wisc.edu/icecube/

[16] E. Aslanides et al., astro-ph/9907432.

[17] L. Trasatti, Nucl. Phys. B (Proc. Suppl.) 70, 442 (1999).

[18] L.V. Volkova, Yad. Fiz. 31, 1510 (1980) [Sov. J. Nucl. Phys. 31, 784 (1980)].

[19] M. Honda, T. Kajita, K. Kasahara, and S. Midorikawa, Phys. Rev. D 52, 4985 (1995).

[20] V. Agrawal, T.K. Gaisser, P. Lipari, and T. Stanev, Phys. Rev. D 53, 1314 (1996).
[21] R. Gandhi, C. Quigg, M.H. Reno, and I. Sarcevic, Phys. Rev. D 58, 093009 (1998).

[22] P. Lipari, M. Lusignoli, and F. Sartogo, Phys. Rev. Lett. 74, 4384 (1995).

[23] Particle Data Group, D.E. Groom et al., Eur. Phys. J. C 15, 171 (2000).

[24] R. Gandhi, C. Quigg, M.H. Reno, and I. Sarcevic, Astropart. Phys. 5, 81 (1996).

[25] T. Gaisser, Cosmic Rays and Particle Physics (Cambridge University Press, Cambridge, England, 1990).

[26] ANTARES web page, http://antares.in2p3.fr/, see, for example, the ANTARES proposal and related publications.

[27] K. Dick, M. Freund, P. Huber, and M. Linder, Nucl. Phys. B598, 543 (2001).

[28] C. Carloganu, Europhysics Neutrino Oscillation Workshop (NOW 2000), Otranto, Italy, 2000.

[29] Y. Oyama, Talk given at the YITP Workshop on Flavor Physics, Kyoto, Japan, 1998, hep-ex/9803014; S. Boyd, Nucl. Phys. B (Proc. Suppl.) 98, 175 (2001).

[30] D.A. Petyt, Yad. Fiz. 63, 1199 (2000) [Phys. At. Nucl. 63, 1122 (2000)].

[31] P. Antonioli, Europhysics Neutrino Oscillation Workshop (NOW2000), Otranto, Italy, 2000, hep-ex/0101040; MONOLITH Proposal — CERN/SPSC 2000-031.

[32] C.K. Jung, hep-ex/0005046.

[33] $\theta_{Z}=0$ degree corresponds to upward going neutrinos and $\theta_{Z}$ $=180^{\circ}$ corresponds to downward going neutrinos.

[34] Although Volkova [18] uses a lower limit of $10^{2} \mathrm{GeV}$ for this approximation, the extrapolation down to $10 \mathrm{GeV}$ is in very good agreement with the flux for lower energies which can be found in Table II of her paper. 